UB-ECM-PF-12-74, ICCUB-12-157

\title{
Non-linear Realizations, Goldstone bosons of broken Lorentz rotations and effective actions for p-branes
}

\author{
Joaquim Gomis $^{a}$ and Kiyoshi Kamimura ${ }^{b}$, and Josep M. Pons ${ }^{a}$ \\ a Departament d'Estructura i Constituents de la Matèria and Institut de Ciències del \\ Cosmos, Universitat de Barcelona, Diagonal 647, 08028 Barcelona, Spain \\ ${ }^{b}$ Department of Physics, Toho University, Funabashi, Chiba 274-8510, Japan \\ E-mails: gomis@ecm.ub.es,kamimura@ph.sci.toho-u.ac.jp, pons@ecm.ub.es,
}

\begin{abstract}
We consider the non-linear realizations of the Poincare group for $p$-branes with local subgroup $S O(1, p) \times S O(D-(p+1))$. The Nambu-Goto $p$-brane action is constructed using the Maurer Cartan forms of the unbroken translations. We perform a throughout phase space analysis of the action and show that it leads to the canonical action of a $p$-brane. We also construct some higher order derivative terms of the effective $p$-brane action using the MC forms of the broken Lorentz transformations.
\end{abstract}

keywords: Non-linear Realizations, p-branes, Space-Time Symmetries, Effective Actions 


\section{Introduction}

Recently there has been a renewal of interest to construct the effective string theory action [1] (see also [2]) using non-linear Lorentz invariance. The lowest order term in derivatives is the well known Nambu-Goto (NG) action. In [3], and references therein, it has been examined the next order Lorentz invariant corrections to the NG action in the static gauge ${ }^{1}$. In that approach it is required the invariance under the broken Lorentz transformations of coordinates of the string, which are the Goldstone bosons associated with the broken translations. The broken Lorentz transformations rotate the longitudinal and transverse directions of the string. In this paper we consider $p$-brane diffeomorphism (Diff) invariant actions in the non-linear realization (NLR) approach [6], see also [7]. In our case they contain the Goldstone bosons of both broken and unbroken translations and Lorentz rotations. The MC forms, pullbacked to the world volume, are used to build these Lagrangians with the geometric tools of the wedge product, Hodge operator, and covariant differentiation. These actions are functionals of both of the embedding variables $x$ and the Lorentz variables $\Phi$.

The general action will be

$$
S[x, \Phi]=\int e[x, \Phi] \mathcal{L}[x, \Phi],
$$

where the volume form $e[x, \Phi]$ is constructed by using the Maurer Cartan (MC) forms associated to unbroken translations. With $\mathcal{L}=1$, it leads to the NG action for the embedding variables, see for example [8][9][10][11], once the Inverse Higgs (IH) mechanism [12], or equivalently the equations of motion (EOM) of the Lorentz variables are used ${ }^{2}$. In this lowest order case in derivatives, the Lorentz variables are non-dynamical.

To construct more general actions with $\mathcal{L} \neq 1$ we can use the MC forms associated to the broken and the unbroken Lorentz transformations. In this case the Lorentz variables are dynamical. Note that the form of the invariant scalar Lagrangian has no additional restrictions, and there is an infinite arbitrariness of choices. For $p$-brane actions we should also consider WZ terms $\mathcal{L}_{W Z}$ that are constructed from closed invariant $\mathrm{p}+2$ forms, $\Omega_{p+2}=d \mathcal{L}_{W Z}$.

If we want to obtain a Lagrangian depending on the embedding coordinates $x$ 's alone, we should express the Lorentz variables in terms of these coordinates. A standard procedure is to impose an invariant set of constraints, so called the IH mechanism (see (1.7)) and to construct the corresponding MC forms on the world volume associated to the broken Lorentz transformations and to the unbroken rotation (to build covariant derivatives) and use them to construct the polynomial and non-polynomial invariants of the coefficients of these forms along the lines of, for example [7]. This procedure give all possible local terms in the effective action.

In this paper we explore a possible different method to express the Lorentz variables in terms of geometrical quantities by solving the equations of motion perturbatively. A starting Lagrangian is called the seed Lagrangian in the following sense: since we want to obtain a Lagrangian depending on the embedding coordinates $x$ 's alone, we should express the Lorentz variables in terms of these variables. This can be

\footnotetext{
${ }^{1}$ See also [4],[5] for recent work on effective strings in the static gauge.

${ }^{2}$ For a recent discussion about the relation between the IH mechanism and the equation of motion of non-dynamical Goldstone bosons see [13].
} 
done by solving perturbatively the equations of motion of the Lorentz variables and substitute these perturbative solutions into the seed Lagrangian. Thus from a seed Lagrangian we obtain a perturbative series of Lagrangians in terms of the embedding variables. Let us insist however that there is an infinite freedom of choosing the seed Lagrangian. Obviously we could limit ourselves to polynomial seed Lagrangians, organized in number of derivatives, as is done in effective theories.

We first illustrate it here in the case of a particle (see details in the section 3 ). We consider a coset $G / H=\left(\right.$ Poincare) $/ S O(D-1)$ and the coset element $g=g_{0} U$, where $g_{0}=e^{i x^{0} P_{0}} e^{i x^{a^{\prime}} P_{a^{\prime}}}$ is the coset representing the Minkowski space and $U=e^{i \theta_{0} b^{\prime}} J^{0}{ }_{b^{\prime}}$ is a general Lorentz rotation, $x^{a^{\prime}}\left(a^{\prime}=1, \ldots D-1\right)$ are the Goldstone bosons of broken translations, $x^{0}$ is the Goldstone boson of the unbroken time translation ${ }^{3}$ and $U$ is parametrized by the Goldstone bosons of the broken Lorentz transformations. The MC forms are given by, $(\underline{a}=0, \ldots, D-1)^{4}$,

$$
\Omega=-i g^{-1} d g=L^{\underline{a}} P_{\underline{a}}+\frac{1}{2} L_{\underline{a}}^{\underline{b}} J^{\underline{a}} \underline{\underline{b}}, \quad L^{\underline{b}}=d x^{\underline{a}} \Phi_{\underline{a}}^{\underline{b}}, \quad L^{\underline{a b}}=\left(\Phi^{-1} d \Phi\right) \stackrel{a b}{ },(1
$$

where $\Phi_{\underline{a}} \underline{b}(\theta)$ is a Lorentz boost parametrized by $\theta_{0}{ }^{b^{\prime}}$. The action ${ }^{5}$ of a free massive particle is the $S O(D-1)$ invariant one form $L^{0}$ associated to the unbroken translation $P_{0}$,

$$
I=-m \int\left(L^{0}\right)^{*}=-m \int d \tau \dot{x}^{\underline{a}} \Phi_{\underline{a}}{ }^{0}(\theta)=-m \int d \tau e .
$$

This action is invariant under Diff, as is the case for every action built from the pullback of $\mathrm{MC}$ forms and with the tools mentioned above. If we compute the momenta

$$
p_{\underline{a}}=\frac{\partial \mathcal{L}}{\partial \dot{x} \underline{a}}=-m \Phi_{\underline{a}^{0}}
$$

therefore $\Phi_{\underline{a}}{ }^{0}(\theta)$ are functions of the momenta of the particle. This fact implies that the Goldstone bosons of broken rotations $\theta_{0}{ }^{b^{\prime}}$ are actually phase space degrees of freedom of the particle.

Now if we regard $\Phi_{\underline{a}}{ }^{0}$ as independent degrees of freedom we can rewrite the action as

$$
I=-m \int d \tau\left(\dot{x}^{\underline{a}} \Phi_{\underline{a}}{ }^{0}+\frac{\gamma}{2}\left(\eta^{\underline{a b}} \Phi_{\underline{a}}{ }^{0} \Phi_{\underline{b}}{ }^{0}+1\right)\right)=\int d \tau\left(p_{\underline{a}} \dot{x}^{\underline{a}}-\frac{\gamma}{2 m}\left(\eta \underline{a b} p_{\underline{a}} p_{\underline{b}}+m^{2}\right)\right),
$$

where $\gamma$ is the Lagrange multiplier to constrain $\Phi_{\underline{a}}{ }^{0}$ to be a time-like unit Lorentz vector. It is the canonical action of the relativistic free particle, as can be extracted from [15] [16]. Therefore the NLR action that include the Goldstone bosons of the translations and the Goldstone bosons associated to the broken boost is the canonical action of the massive relativistic particle.

If we use the equations of motion of $\Phi_{\underline{a}}{ }^{0}$ and $\gamma$, we obtain

$$
\Phi_{\underline{a}}^{0}=-\frac{\dot{x}_{a}}{\sqrt{-\dot{x}^{2}}}
$$

\footnotetext{
${ }^{3}$ The unbroken Lorentz translation $P_{0}$ generates via a right action [14][11] a transformation which is equivalent to the world-line diffeomorphism.

${ }^{4}$ We follow the notations of [10].

${ }^{5}$ In writing the Lagrangians, the pullback to the world volume of the MC forms is always understood. The pullback notation * is sometimes suppressed just for notational simplicity.
} 
Note that $\Phi_{\underline{a}}{ }^{a^{\prime}}$ is normal to the worldline and is equivalent to the vanishing the MC form $L^{a^{\prime}}$ associated to the broken translations

$$
L^{a^{\prime}}=d x^{\underline{a}} \Phi^{a^{\prime}}=0
$$

which is known as the inverse Higgs (IH) mechanism [12].

We can construct corrections to the NG action of the particle by considering the pullback of the $\mathrm{MC}$ forms associated to the broken Lorentz transformations ${ }^{6}$. We use the standard tools (wedge product, Hodge operator, and covariant differentiation) available with the MC forms. In the case of the particle we define the Hodge operator by assigning $L^{0}=d \tau e$ as the volume form which implies $* d \tau=-e^{-1}$. Since we want corrections to the NG action in the spirit of effective theories, we first consider the term with first order derivatives of the Lorentz variables. In particular the polynomial one, which is quadratic in the velocities, whereas the coefficient is chosen to be small. This is made by choosing, among the $S O(D-1)$ invariant one forms, the structure $L_{0 a^{\prime}} * L^{0 a^{\prime}}$, which depends at most on the velocities $\dot{\Phi}^{\underline{a b}}$. Adding this term to the action (1.5) we obtain

$$
I=-m \int d \tau\left\{e+\frac{\gamma}{2}\left(\Phi_{\underline{a}}^{0} \Phi^{\underline{a} 0}+1\right)+\frac{\beta}{2 m^{2}}\left(\frac{1}{e} \dot{\Phi}^{\underline{a}}{ }_{0}\left(\eta_{\underline{a b}}-\Phi_{\underline{a} 0} \Phi_{\underline{b}}^{0}\right) \dot{\Phi}^{\underline{b} 0}\right)\right\},
$$

or better, with a redefinition of $\gamma$,

$$
I=-m \int d \tau\left\{e+\frac{\gamma}{2}\left(\Phi_{\underline{a}^{0}} \Phi^{\underline{a} 0}+1\right)+\frac{\beta}{2 m^{2}}\left(\frac{1}{e} \dot{\Phi}^{\underline{a}}{ }_{0} \dot{\Phi}^{\underline{a} 0}\right)\right\}
$$

where the constant parameters $\beta$ is dimensionless and the term with $\beta$ is considered as a perturbation of the free particle action ${ }^{7}$. Notice that in this Lagrangian the $\Phi_{\underline{b}}{ }^{0}$ variables are independent dynamical variables. We have been unable to solve dynamically to all orders in $\beta$ the equation of motion of $\Phi_{\underline{b}}{ }^{0}$ in terms of $x^{\underline{a}}, \gamma$.

We do not use other possible Lagrangian candidates, like $L_{a^{\prime}} * L^{0 a^{\prime}}$ or $L_{a^{\prime}} * L^{a^{\prime}}$, since they vanish at lowest order due to (1.7). We could also add invariant terms with an increasing number of derivatives of $\Phi$ like for instance $\left(\tilde{D} * L_{0 a^{\prime}}\right) *\left(\tilde{D} * L_{0 a^{\prime}}\right)$, where $\tilde{D}$ is the covariant differential, as in effective theories. We will focus in this paper the lowest order in derivatives, and we will use this action (1.9) as a seed to produce a set of geometrical invariant actions. In fact in order to make contact with an effective Lagrangian written in terms of the geometric quantities of the world-line in Minkowski space we use the procedure of solving the equations of motion of $\Phi_{\underline{b}}{ }^{0}$ perturbatively in $\beta$, in terms of the velocities, accelerations, $\ldots$ of the particle. At lowest order in $\beta, \Phi_{\underline{b}}{ }^{0}$ is given by (1.6) and the geometrical action becomes ${ }^{8}$, in terms of proper time $s$,

$$
I=-m \int d s\left(1+\frac{\beta}{2 m^{2}} \kappa_{1}^{2}+O\left(\beta^{2}\right)\right)
$$

\footnotetext{
${ }^{6} \mathrm{In}$ [17] [18] the MC forms associated to broken Lorentz rotations were used to construct relativistic gauge fixed (super) particle action in two dimensions with extrinsic curvature.

${ }^{7}$ We could have also added terms with higher derivatives in $\Phi$. We will not consider those terms in this paper.

${ }^{8}$ If we only consider the term of order $\beta$ the dynamics of that Lagrangian is not equivalent to the starting Lagrangian (1.9). The equivalence would only be achieved with the full perturbative series.
} 
where $\kappa_{1}$ is the first curvature of the world-line, $\kappa_{1}^{2}=(A)^{2}, A \underline{a}=\frac{d^{2} x \underline{a}}{d s^{2}}$. Note that we do not make use of the EOM of the coordinates $x$, unlike what is customarily done in the standard procedure for effective field theories [2][3]. At first order in $\beta$ the solution for $\Phi_{\underline{b}}^{0}$ from (1.9) departs from the IH mechanism,

$$
\Phi_{\underline{a}}^{0}=-U_{\underline{a}}-\beta \Sigma_{\underline{a}}
$$

where $U_{\underline{a}}=\frac{d x_{\underline{a}}}{d s}$ and $\Sigma_{\underline{a}}=\frac{d^{3} x_{\underline{b}}}{d s^{3}}\left(\delta_{\underline{b}} \underline{a}+U_{\underline{b}} U \underline{a}\right)$ is the relativistic jerk vector, see for example [19] [20]. Note that there is a rationale for the presence of the relativistic jerk vector, since it is orthogonal to the velocity, and this orthogonality is already imposed on $\Phi_{\underline{a}}{ }^{0}$ in (1.11) due to the Lorentz constraints $\eta^{\underline{a b}} \Phi_{\underline{a}}{ }^{0} \Phi_{\underline{b}}{ }^{0}+1=0$.

By substituting (1.11) into (1.9) we can consider the expansion of the action up to order $\beta^{2}$ in terms of the embedding variables,

$$
I_{2}=m \int d s \frac{\beta^{2}}{2 m^{4}}\left(\kappa_{1}^{2} \kappa_{2}^{2}+\left(\frac{d \kappa_{1}}{d s}\right)^{2}\right),
$$

where $\kappa_{2}^{2}=\left(\frac{d^{3} x}{d s^{3}} O_{2} \frac{d^{3} x}{d s^{3}}\right) / \kappa_{1}^{2}$ is the second curvature of the world-line, $\left(O_{2}\right)$ is the projector orthogonal to accelerations and velocities ( $s$ is proper time). We should notice that the combination of curvatures $\kappa_{1}^{2} \kappa_{2}^{2}+\left(\frac{d \kappa_{1}}{d s}\right)^{2}$ appearing at order $\beta^{2}$ of the effective Lagrangian (1.12) is the $\Sigma^{2}$ of the relativistic jerk vector $\Sigma_{\underline{a}}$.

Note that we could consider within our approach more general, non-polynomial, actions, like

$$
\mathcal{L}=-e\left(1+\frac{\gamma}{2}\left(\Phi^{2}+1\right)\right)+\frac{\tilde{\beta}}{2} \sqrt{(\dot{\Phi})^{2}}
$$

In this case the relation to order $\tilde{\beta}$ among the Lorentz variables and the geometrical quantities is given

$$
\Phi^{\underline{a}} 0=-U^{\underline{a}}+\frac{\tilde{\beta}}{2 \sqrt{-\dot{x}^{2}}} \frac{A^{\underline{a}}}{\sqrt{(A)^{2}}}
$$

As long as the seed Lagrangian contains the term with first time derivatives of the Lorentz variables, the first correction term to the NG will be a functional of the first extrinsic curvature.

In this paper we will see that the previous results for the particle are extended to the case of a $p$-brane and show that:

1. The Goldstone bosons of broken Lorentz rotations are functions of the phase space degrees of freedom of the $p$-brane.

2. The NLR action constructed from the MC forms of unbroken translations leads in a natural way to the canonical form of NG action. Since the Goldstone bosons of the broken rotations are non-dynamical we can eliminate them through their own equations of motion and one gets the world-volume NG action [10].

3. We can also construct a WZ term for a $p$-brane in dimensions $p+2$ using only the MC forms of the translations ${ }^{9}$.

\footnotetext{
${ }^{9}$ For the case of the string see $[21][22]$ and for the 2-brane [23].
} 
4. As in (1.5) it is often useful to introduce also Goldstone bosons associated to the unbroken Lorentz rotations as independent degrees of freedom without using explicit parametrization of the Lorentz transformation. In this case the Poincare invariant $p$-brane action will be invariant under local $S O(1, p) \times S O(D-(p+1))$ rotations as well as world-volume diffeomorphism (Diff).

5. We also consider terms of the effective action of a p-brane with non-linearly realized Poincare symmetry by adding invariant forms associated with the broken Lorentz transformations. In these cases actions, like the particle case, will contain the first derivatives of the Goldstone bosons of broken rotations and the $\Phi$ 's become dynamical. The Lagrangian with both the embedding variables and the Lorentz variables is used as a seed to produce corrections, in terms of the embedding variables, to the NG Lagrangian. The procedure consist in solving for the Lorentz variables by using the equations of motion (EOM) iteratively and substituting them back into the Lagrangian so that it will produce an effective action in terms of geometrical quantities, like the intrinsic curvature, extrinsic curvature and the higher curvatures of the world-volume.

The organization of the paper is as follows. In section 2 we will construct the action of a tensionfull $p$-brane with the lowest order derivatives using the non-linear realization approach with local subgroup $S O(1, p) \times S O(D-(p+1))$. We will perform the Hamiltonian-Dirac analysis that leads to the canonical action of the NG action. In section 3 we will construct leading order -and beyond- corrections to the NG action. Finally we will present some conclusions and outlook. There are two appendices with technical details.

Note added: when this paper was in a process of submission it has appeared a paper by Gliozzi and Meineri [24] which proposes a different procedure to construct some higher order derivative corrections to the Nambu Goto action. Their results agree with ours.

\section{Nonlinear realization with local subgroup $S O(1, p) \times S O(D-(p+1))$}

In this section we will construct the $p$-brane action using the NLR approach [11] by Callan, Coleman, Wess and Zumino [6]. However we will not follow the method in terms of coset representatives. In the present case it is more useful to reformulate the theory of non-linear realizations in terms of the group elements ${ }^{10}$ of $G$ themselves rather than those of the coset $G / H$. We consider group elements $g$ of $G$ and take the symmetries of the non-linearly realized theory given by

$$
g(x) \rightarrow g_{0} g(x) \text { and } g(x) \rightarrow g(x) h(x)
$$

where $g_{0} \in G$ is a rigid transformation while the second independent local transformation $h(x)$ is an arbitrary space-time dependent transformation that belongs to $H$. In this more general way the theory contains gauge degrees of freedom that can be

\footnotetext{
${ }^{10}$ For the case of internal symmetries see [25].
} 
fixed using the local $H$ transformations. We will refer to such a formulation as a non-linear realization of a group $G$ with local subgroup $H$.

The global $G$ transformations of the coset coordinates $x$ 's are determined up to the local $H$ transformations and are in general non-linear. One could from the beginning use these local $H$ transformations to fix the gauge freedom and so work only with coset representatives. This is equivalent to the original approach and it requires the $H$-compensating transformations for the global $g_{0}$ transformations. One can also work in a half way house where only some of the fields are removed.

For the case of relativistic $p$-branes we consider the NLR of $G=I S O(D-1,1)$ with the local subgroup $S O(1, p) \times S O(D-(p+1))$. The generators ${ }^{11} P_{a}, J^{a}{ }_{b}, J^{a^{\prime}}{ }_{b^{\prime}}$ generate the unbroken translations, local longitudinal and transverse rotations via the right transformations [14]. In the non-linear realization the reparametrization invariance corresponds to the invariance under unbroken translations with additional local Lorentz rotations for a general $p$-brane, see [11].

The group elements are parametrized by

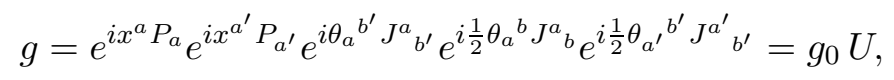

where $g_{0}=e^{i x^{a} P_{a}} e^{i x^{a^{\prime}} P_{a^{\prime}}}$ is the coset representing the Minkowski space and $U$ is a general element of the Lorentz group. In $(2.2)$ we have introduced $S O(1, p) \times S O(D-(p+1))$ degrees of freedom in addition to the coset elements of $\frac{I S O(D-1,1)}{S O(1, p) \times S O(D-(p+1))}$ used for example in [10]. The MC form is given by

$$
\Omega=-i g^{-1} d g=L^{\underline{a}} P_{\underline{a}}+\frac{1}{2} L_{\underline{a}}^{\underline{b}} J^{\underline{a}} \underline{\underline{b}},
$$

where the vielbein and spin connection forms in the flat $D$ dimensional target space are

$$
L^{\underline{a}}=d x^{\underline{b}} \Phi_{\underline{\underline{b}}} \underline{\underline{a}}, \quad L^{\underline{a}} \underline{\underline{b}}=\Phi_{\underline{c}}^{\underline{a}} d \Phi^{\underline{c}} \underline{\underline{b}},
$$

and are satisfying the $\mathrm{MC}$ equations

$$
d L^{\underline{a}}+L^{\underline{a}} \underline{\underline{b}} \wedge L^{\underline{b}}=0, \quad d L^{\underline{a b}}+L^{\underline{a}} \underline{\underline{c}} \wedge L^{\underline{c b}}=0 .
$$

Here $\Phi_{\underline{b}} \underline{a}$ is a finite Lorentz transformation parametrized by $\theta_{a}{ }^{b^{\prime}}, \theta_{a}{ }^{b}, \theta_{a^{\prime}}{ }^{b^{\prime}}$. The explicit form of the finite Lorentz transformation $\Phi$ in terms of the Goldstone fields $\theta$ is not simple. Also, the local $S O(1, p) \times S O(D-(p+1))$ transformations of $\theta$ are involved.

Like in the case of the particle (1.5), it is more convenient to consider the $D^{2}$ elements of $\Phi_{\underline{a}} \underline{b}$ of the Lorentz transformation themselves as new variables restricted by the orthonormality constraint

$$
C \stackrel{c d}{c}=\eta^{\underline{a b}} \Phi_{\underline{a}}^{\underline{c}} \Phi_{\underline{b}} \underline{d}-\eta \underline{\underline{c d}}=0,
$$

and with the implicit choice of $\operatorname{det} \Phi=1, \Phi_{0}{ }^{0} \geq 1$. Under the local rotations $O_{b}{ }^{a} \in$ $S O(1, p)$ and $O_{b^{\prime}}{ }^{\prime} \in S O(D-(p+1))$ it transforms as

$$
\Phi_{\underline{a}}{ }^{a} \rightarrow \Phi_{\underline{a}^{b}}{ }^{b}{ }_{b}{ }^{a}(\xi), \quad \Phi_{\underline{a}}{ }^{a^{\prime}} \rightarrow \Phi_{\underline{a}}{ }^{b^{\prime}} O_{b^{\prime}}{ }^{a^{\prime}}(\xi),
$$

\footnotetext{
${ }^{11} \underline{a}$ goes over all possible values from 0 to $D-1$ while the unprimed indices $a$ take the values $a=0, \ldots, p$ and primed indices $a^{\prime}$ take the values $p+1, \ldots, D-1$. The latter are the indices which are longitudinal and transverse to the brane respectively.
} 
where $\xi^{i},(i=0,1, \ldots, p)$ are the $p+1$ coordinates of the world-volume of a $p$-brane. The number of Goldstone bosons $\theta$ of $(2.2)$ is $\frac{D(D-1)}{2}$. We describe them by the $D^{2}$ elements of $\Phi_{\underline{a}}^{\underline{b}}$ subject to the $\frac{D(D+1)}{2}$ conditions $(2.6)$. $\frac{(p+1) p}{2}$ of them are the gauge degrees of freedom of $S O(p, 1)$ and $\frac{(D-(p+1))((D-(p+2))}{2}$ are the ones associated to the local $S O(D-(p+1))$. So the number of non-gauge degrees of freedom for the $\Phi$ variables will be $(p+1)(D-(p+1))$ which agrees with the counting of Goldstone bosons $\theta_{a}{ }^{\prime}$ associated to broken Lorentz rotations of the coset $\frac{I S O(D-1,1)}{S O(1, p) \times S O(D-(p+1))}$ [10].

\subsection{NG $p$-brane Lagrangian}

The action of relativistic $p$-branes with lowest order of derivatives is constructed from the pullback of the MC forms (2.4) on the world-volume, with dimensionless coordinates $\left(\xi^{0}, \xi^{m}\right), m=1, \ldots, p$. It must be invariant under world-volume diffeomorphism associated to the longitudinal unbroken translation invariance and local longitudinal and transverse Lorentz rotations. The Lagrangian density, for a generic dimension, is given by

$$
\mathcal{L}=\mathcal{L}_{0}+\mathcal{L}_{1}
$$

with $\mathcal{L}_{0}$ is the world-volume density of the $p+1$-brane ${ }^{12}$

$$
d^{p+1} \xi \mathcal{L}_{0}=\kappa \frac{1}{(p+1) !} \epsilon_{a_{0} \ldots a_{p}} e^{a_{0}} \ldots e^{a_{p}}=-d^{p+1} \xi \kappa e,
$$

where $\kappa$ is the tension of the $p$-brane with dimension $[\kappa]=m^{p+1}$ and $e=\operatorname{det}\left(e_{i}{ }^{a}\right)$ with $(p+1)$-bein given by

$$
e^{a}=\left(L^{a}\right)^{*}=d \xi^{i} e_{i}^{a} \quad e_{i}^{a}=\partial_{i} x^{\underline{a}} \Phi_{\underline{a}}^{a}
$$

which is the pullback of the $L^{a}$ in $(2.4) . \mathcal{L}_{1}$ is the Lagrange multiplier term for the orthonormality of the $D$-beins $\Phi_{\underline{a}} \underline{\underline{c}},(2.6)$,

$$
\mathcal{L}_{1}=e \frac{\gamma_{\underline{c d}}}{2}\left(\eta^{\underline{a b}} \Phi_{\underline{a}}^{\underline{c}} \Phi_{\underline{\underline{b}}}^{\underline{d}}-\eta \underline{\underline{c d}}\right) .
$$

We can write the Lagrangian $(2.8)$ as $^{13}$

$$
\mathcal{L}=-\kappa^{\prime} e
$$

with

$$
\kappa^{\prime}=\kappa-\frac{\gamma_{\underline{c d}}}{2}\left(\eta^{\underline{a b}} \Phi_{\underline{a}}^{\underline{c}} \Phi_{\underline{b}} \underline{\underline{d}}-\eta^{\underline{c d}}\right) .
$$

Not all of the Euler-Lagrange (EL) equations of motion are independent but there are $p+1$ Noether identities for diffeomorphism invariance,

$$
\frac{\delta \mathcal{L}}{\delta x^{\underline{a}}} \partial_{i} x^{\underline{a}}+\frac{\delta \mathcal{L}}{\delta \Phi_{\underline{b}} \underline{a}} \partial_{i} \Phi_{\underline{b}} \underline{a}+\frac{\delta \mathcal{L}}{\delta \gamma_{\underline{b a}}} \partial_{i} \gamma_{\underline{b a}} \equiv 0, \quad(i=0,1, \ldots, p) .
$$

\footnotetext{
${ }^{12}$ We often omit the $\wedge$ symbol for the wedge products.

${ }^{13}$ A similar action in terms of Lorentz harmonics was considered in [26] [27].
} 
The Noether identities associated with the local $S O(p, 1) \times S O(D-(p+1))$ gauge invariance are $^{14}$

$$
\frac{\partial \mathcal{L}}{\partial \Phi_{\underline{c}}[a} \Phi_{\underline{c} b]}+2 \frac{\partial \mathcal{L}}{\partial \gamma_{\underline{c}}[a} \gamma_{\underline{c} b]} \equiv 0, \quad \frac{\partial \mathcal{L}}{\partial \Phi_{\underline{c}}^{\left[a^{\prime}\right.}} \Phi_{\left.\underline{c} b^{\prime}\right]}+2 \frac{\partial \mathcal{L}}{\partial \gamma_{\underline{c}}\left[a^{\prime}\right.} \gamma_{\left.\underline{\underline{c}} b^{\prime}\right]} \equiv 0 .
$$

They are $\frac{p(p-1)}{2}+\frac{(D-(p+1))(D-p-2)}{2}$ relations among these equations of motion.

The presence of gauge symmetries and their associated Noether identities results in the appearance of the first class constraints in the Hamiltonian formalism. In addition, since the variables $\Phi_{\underline{b}} \underline{\underline{a}}, \gamma_{\underline{b a}}$ are non-dynamical (for the Lagrangian (2.8)), the EL equations will produce Lagrange constraints (relations among coordinates and velocities). As we will see they will produce Hamiltonian secondary constraints and relations among the arbitrary functions appearing in the Hamiltonian.

Now we will make contact with ordinary NG action written in terms of the coordinates of the p-brane. In fact, if we introduce the inverse vielbein $e_{b}{ }^{i}, e_{b}{ }^{i} e_{i}{ }^{a}=\delta^{a}{ }_{b}$, the IH mechanism $L^{a^{\prime}}=0$ like the particle case (1.7) and the orthonormality condition (2.6), we can write $\Phi \underline{a}_{b}$ as

$$
\Phi^{\underline{a}}{ }_{b}=e_{b}^{i} \partial_{i} x^{\underline{a}},
$$

we have also

$$
e_{i}^{b}=\eta^{b c} e_{c}^{j} \bar{g}_{j i}
$$

where $\bar{g}_{i j}$ is the induced metric, $\bar{g}_{i j}=\left(\partial_{i} x_{\underline{a}} \partial_{j} x^{\underline{a}}\right)$. Taking the determinant of $(2.17)$ we have that the Lagrangian (2.12) becomes

$$
\mathcal{L}=-\kappa \sqrt{-\bar{g}}
$$

which is the ordinary NG action.

\section{$2.2 \quad$ WZ Lagrangian density}

Apart from the invariant Lagrangian density (2.8) valid for any dimension we can construct a WZ term for a $p$-brane in dimensions $D=p+2$ using only the MC forms of the translations ${ }^{15} L^{\underline{a}}$. In fact

$$
\Omega_{p+2}=\epsilon_{\underline{a}_{0} \cdots \underline{a}_{p+1}} L^{\underline{a}_{0}} \wedge \cdots L^{\underline{a}_{p+1}}=(\operatorname{det} \Phi) \epsilon_{\underline{a}_{0} \cdots \underline{a}_{p+1}} d x^{\underline{a}_{0}} \wedge \cdots d x^{\underline{a}_{p+1}}
$$

is a closed invariant $(p+2)$ form. Since $\operatorname{det} \Phi=1$ we can write it as

$$
\Omega_{p+2}=d\left(\epsilon_{\underline{a}_{0} \cdots \underline{a}_{p+1}} x^{\underline{a}_{0}} \wedge d x^{\underline{a}_{1}} \wedge \cdots d x^{\underline{a}_{p+1}}\right)=d\left(L_{W Z}\right) .
$$

We can either add $L_{W Z}$ on the NG Lagrangian [21] [22] or consider the $p+1$ form $L_{W Z}$ solely as a particle model Lagrangian. The WZ term of the $p$-brane action represents the coupling of the $p$-brane to a $(\mathrm{p}+1)$ form Abelian gauge potential given by $A_{p+1}=\epsilon_{\underline{a}_{0} \cdots \underline{a}_{p+1}} x^{\underline{a}_{0}} \wedge d x^{\underline{a}_{1}} \wedge \cdots d x^{\underline{a}_{p+1}}$, this coupling breaks parity invariance for odd $p$.

\footnotetext{
${ }^{14}$ Our convention is $\frac{\delta \gamma_{\underline{a} b}}{\delta \gamma_{\underline{c}}}=\frac{1}{2}\left(\delta \underline{\underline{a}} \delta \underline{\underline{b}}+\delta_{\underline{b}}^{\frac{c}{b}} \delta \underline{\underline{a}}\right)$.

${ }^{15}$ See [21][22] for the case of the string and [23] for the 2-brane.
} 


\subsection{NLR action versus canonical action}

In this subsection we will see how the Goldstone bosons of the broken Lorentz generators are functions of the phase space variables of the $p$-brane. This result generalizes (1.4) of the particle, sketched in the introduction to the $p$-brane case. We will see that the NLR action (2.8) leads in a natural way to the canonical action of a $p$ brane. The definition of all the canonical momenta from the action $(2.8)^{16}$ gives primary constraint equations (See the details of the Hamiltonian formalism in the appendix B)

$$
\begin{aligned}
\phi_{\underline{a}} & \equiv p_{\underline{a}}+\kappa^{\prime} e e_{b}^{0} \Phi_{\underline{a}}{ }^{b}=0, \\
\phi^{\underline{a}} \underline{b} & \equiv \Pi \underline{a}_{\underline{b}}=0, \\
\phi \frac{a b}{\gamma} & \equiv p \frac{a b}{\gamma}=0 .
\end{aligned}
$$

Note that the combination $e e_{b}^{0}$ does not depend on the velocities ${ }^{17} \dot{x}^{\underline{a}}$. The Hamiltonian is a sum of primary constraints,

$$
\mathcal{H}_{D}=\int d^{p} \xi \mathcal{H}, \quad \mathcal{H}=\phi_{\underline{a}} \lambda^{\underline{a}}+\phi \underline{\underline{a}}_{\underline{b}} \Lambda_{\underline{a}} \underline{b}+\phi \frac{a b}{\gamma} \lambda_{\underline{a b}}^{\gamma},
$$

where $\lambda^{\underline{a}}, \Lambda_{\underline{a}} \underline{b}$ and $\lambda_{a \underline{b}}^{\gamma}$ are arbitrary functions of $\xi$ at this moment. Using the Hamilton's equations they are related to the velocities,

$$
\left.\dot{x}^{\underline{a}}=\left\{x^{\underline{a}}, \mathcal{H}_{D}\right\}=\lambda^{\underline{a}}, \quad \dot{\gamma}_{\underline{a} b}=\left\{\gamma_{\underline{a b}}, \mathcal{H}_{D}\right\}=\lambda_{\underline{a b}}^{\gamma}, \quad \dot{\Phi}_{\underline{a}} \underline{b}=\left\{\Phi_{\underline{a}} \underline{b}, \mathcal{H}_{D}\right\}=\Lambda_{\underline{\alpha}} \underline{\underline{b}} 2.25\right)
$$

The Hamilton's equations for momenta reproduce the EL equations. The consistency condition that the primary constraints (2.21)-(2.23) remain zero under time evolution gives the secondary constraints

$$
\begin{gathered}
\chi^{\underline{a b}} \equiv C \frac{a b}{2}=\frac{1}{2}\left(\eta^{\underline{c d}} \Phi_{\underline{\underline{c}}}^{\underline{a}} \Phi_{\underline{d}}^{\underline{b}}-\eta^{\underline{a b}}\right)=0, \\
\gamma_{b^{\prime} c}=\gamma_{b^{\prime} c^{\prime}}=0, \quad \gamma_{a b}-\kappa \eta_{a b}=0, \\
\chi_{m}{ }^{b^{\prime}} \equiv \partial_{m} x^{\underline{a}} \Phi_{\underline{a}}{ }^{b^{\prime}}=0, \quad(m=1, \ldots, p)
\end{gathered}
$$

and conditions on $\lambda^{\underline{a}}$ and $\Lambda$,

$$
\begin{gathered}
\tilde{\lambda}^{b^{\prime}} \equiv \lambda^{\underline{a}} \Phi_{\underline{a}}{ }^{b^{\prime}}=0 \\
\left(e e_{b}{ }^{0} \Lambda_{\underline{c}}^{b}+e e_{b}{ }^{m} \partial_{m} \Phi_{\underline{c}}^{b}\right) \Phi \underline{c}_{a^{\prime}}=0,
\end{gathered}
$$

where $e e_{b}{ }^{m},(m=1, \ldots, p)$ are linear functions of $\lambda^{\underline{a}}=\dot{x}^{\underline{a}}$. Eq.(2.26) is the orthonormality of $\Phi$ and Eq.(2.27) fixes the $\gamma_{\underline{a b}}$. Eq.(2.28) and Eq.(2.29) coincide with the vanishing of the MC forms $L^{b^{\prime}}$ in (2.4) associated to broken translations, which is known as the inverse Higgs mechanism (IH) [12].

The stability of the secondary constraints does not produce any further constraints but only conditions on the multipliers,

$$
\dot{\chi} \underline{\underline{a b}}=\frac{1}{2}\left(\eta \underline{\underline{c d}} \Lambda_{\underline{c}}^{\underline{a}} \Phi_{\underline{d}}^{\underline{b}}+\eta \eta^{\underline{c d}} \Phi_{\underline{\underline{c}}}^{\underline{a}} \Lambda_{\underline{d}}^{\underline{b}}\right)=0
$$

\footnotetext{
${ }^{16}$ We consider $\xi^{0}$ as the canonical time and use "dot" as $\xi^{0}$ derivative. We often do not write the dependence on $\xi^{i}$.

${ }^{17} \mathrm{It}$ is useful the relation $e e_{a}{ }^{i}=-\frac{1}{p !} \epsilon^{i i_{1} \ldots i_{p}} \epsilon_{a a_{1} \ldots a_{p}} e_{i_{1}}{ }^{a_{1}} \ldots e_{i_{p}}{ }^{a_{p}}$.
} 


$$
\dot{\chi}_{\underline{a b}}^{\gamma}=\lambda_{\underline{a b}}^{\gamma}=0
$$

and

$$
\partial_{m} x^{\underline{a}} \Lambda_{\underline{a}}^{b^{\prime}}+\partial_{m} \lambda \underline{a}_{\underline{a}}{ }^{b^{\prime}}=0 .
$$

If we redefine the arbitrary functions $\Lambda_{\underline{a}} \underline{b}$ in terms of $\Omega_{\underline{a}} \underline{b}$ by $\Lambda_{\underline{a}} \underline{\underline{b}} \equiv \Phi_{\underline{a}} \underline{\underline{c}} \Omega_{\underline{\underline{b}}} \underline{\underline{b}}$, the consistency condition (2.31) imposes antisymmetry of $\Omega \underline{a b}$, and Eq.(2.30)-(2.33) are solved for $\Omega_{b}^{b^{\prime}}$ as

$$
{\Omega_{a^{\prime}}}^{b}=\left(\left(e e_{[d}^{m} e_{a]}^{0} \Phi_{\underline{a}}^{a} \partial_{m} \Phi_{\underline{c}}^{d} \Phi^{\underline{c}} a^{\prime}\right) \frac{\left(e e^{b 0}\right)}{\mathbf{g}}-\left(\partial_{m} \Phi_{\underline{a} a^{\prime}}\right) \mathbf{g}^{m \ell} e_{\ell}^{b}\right) \lambda^{\underline{a}}
$$

where $\mathbf{g}^{m \ell}$ is the inverse of the spatial world-sheet induced metric $\mathbf{g}_{m \ell}=e_{m} \underline{a}_{\ell \underline{a}}$ and $\mathbf{g}$ is the determinant of $\mathbf{g}_{m \ell}$. There remain arbitrary anti-symmetric Hamiltonian multipliers $\Omega_{a b}, \Omega_{a^{\prime} b^{\prime}}$ and $\tilde{\lambda}^{a} \equiv \lambda^{a} \Phi_{\underline{a}}{ }^{a}$ corresponding to local $S O(p+1), S O(D-(p+$ 1)) and Diff $p+1$ gauge invariances.

The Hamiltonian is written as a linear combinations of constraints with the independent arbitrary multipliers as

$$
\mathcal{H}=\hat{\lambda}^{\perp} \mathcal{H}_{\perp}+\hat{\lambda}^{m} \mathcal{H}_{m}+\frac{1}{2}\left(J_{a b} \tilde{\Omega}^{b a}+J_{a^{\prime} b^{\prime}} \tilde{\Omega}^{b^{\prime} a^{\prime}}\right),
$$

where we have used the fact that products of two constraints vanish as strong equations. The constraints appearing here are the first class combinations of the constraints $^{18}$

$$
\begin{aligned}
\mathcal{H}_{\perp} & =\frac{1}{2 \kappa}\left(\eta \underline{\underline{a}}_{\underline{b}} p_{\underline{a}} p_{\underline{b}}+\kappa^{2} \mathbf{g}\right)+J^{b a^{\prime}}\left(e e_{[d}^{m} e_{b]}^{0}\right)\left(\partial_{m} \Phi_{\underline{c}}^{d} \Phi^{\underline{c}} a^{\prime}\right) \\
\mathcal{H}_{m} & =p_{\underline{a}} \partial_{m} x^{\underline{a}}+\Pi \underline{\underline{a}}_{\underline{b}} \partial_{m} \Phi_{\underline{a}} \underline{\underline{b}} \\
J_{a b} & =\Pi \underline{c}_{[a} \Phi_{\underline{c} b]} \\
J_{a^{\prime} b^{\prime}} & =\Pi \underline{\underline{c}}_{\left[a^{\prime}\right.} \Phi_{\left.\underline{c} b^{\prime}\right]}
\end{aligned}
$$

and the arbitrary multipliers $\hat{\lambda}^{\perp}, \hat{\lambda}^{m}, \tilde{\Omega}^{b a}, \tilde{\Omega}^{b^{\prime} a^{\prime}}$ are given in (B.61) and (B.73).

The other independent constraints are the second class constraints that are used to reduce the phase space variables. In order to find them explicitly we rewrite the variables $\Phi_{\underline{a}} \underline{b}$ in terms of new variables as

$$
\Phi_{\underline{a}^{\underline{b}}}=\left(\begin{array}{cc}
B_{1 a}{ }^{c} & \tilde{\varphi}_{a}^{c^{\prime}} B_{2 c^{\prime}} d^{\prime} \\
-\varphi^{d}{ }_{a^{\prime}} B_{1 d}{ }^{c} & B_{2 a^{\prime}} d^{\prime}
\end{array}\right)\left(\begin{array}{cc}
\phi_{c}{ }^{b} & 0 \\
0 & \phi_{d^{\prime}} b^{\prime}
\end{array}\right)
$$

where $B_{1}$ and $B_{2}$ are symmetric matrices defined by

$$
\left(\left(B_{1}\right)^{-2}\right)_{a}^{b}=\left(\delta_{a}{ }^{b}+\varphi_{a}{ }^{c^{\prime}} \varphi^{b}{ }_{c^{\prime}}\right), \quad\left(\left(B_{2}\right)^{-2}\right)_{a^{\prime}}{ }^{b^{\prime}}=\left(\delta_{a^{\prime}}{ }^{b^{\prime}}+\tilde{\varphi}_{a^{\prime}}^{c} \tilde{\varphi}_{c}^{b^{\prime}}\right) .
$$

In terms of these variables the orthonormality constraints (2.26) are written as

$$
\phi_{a}{ }^{c} \phi^{b}{ }_{c}=\left(\phi \phi^{T}\right)_{a}{ }^{b}=\delta_{a}{ }^{b}, \quad \phi_{c^{\prime}}{ }^{\prime} \phi_{b^{\prime}}^{c^{\prime}}=\left(\phi^{T} \phi^{\prime}\right)^{a^{\prime}}{ }_{b^{\prime}}=\delta_{b^{\prime}}^{a^{\prime}}, \quad \tilde{\varphi}_{a}{ }^{b^{\prime}}=\varphi_{a}{ }^{b^{\prime}} .
$$

\footnotetext{
${ }^{18}$ Notice that the first class constraint $\mathcal{H}_{\perp}$ depends on the broken rotations $J^{b a^{\prime}}$, this is in agreement with results of [11] about local right translations.
} 
It means $\phi_{a}{ }^{b}$ is a group element of $S O(p, 1)$ and $\phi_{a^{\prime}}{ }^{b^{\prime}}$ belongs to $S O(D-(p+1))$. Using the local invariances we can take $\phi_{a}{ }^{b}=\delta_{a}{ }^{b}, \phi_{a^{\prime}}{ }^{{ }^{\prime}}=\delta_{a^{\prime}}{ }^{b^{\prime}}$. In this gauge the second class constraints are $\tilde{\varphi}_{b^{\prime}}^{a}=\varphi_{b^{\prime}}^{a}$ and $\varphi_{a}{ }^{b^{\prime}}$ are expressed in terms of $p_{\underline{a}}$ and $\partial_{m} x^{\underline{a}} \operatorname{as}^{19}$

$$
\begin{aligned}
\varphi_{a_{0}}{ }^{b^{\prime}} & =\left.\frac{-1}{p ! \Delta} \epsilon^{i_{0} \ldots i_{p}} \epsilon_{a_{0} \ldots a_{p}} \partial_{i_{1}} x^{a_{1}} \ldots \partial_{i_{p}} x^{a_{p}} \partial_{i_{0}} x^{b^{\prime}}\right|_{\dot{x} \underline{a} \rightarrow p \underline{a}}, \\
\Delta & =\left.\frac{-1}{(p+1) !} \epsilon^{i_{0} \ldots i_{p}} \epsilon_{a_{0} \ldots a_{p}} \partial_{i_{0}} x^{a_{0}} \partial_{i_{1}} x^{a_{1}} \ldots \partial_{i_{p}} x^{a_{p}}\right|_{\dot{x} \underline{a} \rightarrow p \underline{a}},
\end{aligned}
$$

where the replacement $\partial_{0} x^{\underline{a}} \rightarrow p^{\underline{a}}$ is to be done in the right hand side of the previous equations.

Therefore the Goldstone bosons $\varphi_{a}{ }^{b^{\prime}}$ associated to Lorentz broken rotations are written as functions of the phase space variables of the $p$-brane. Now all second class constraints are used to reduce the phase space to that of the space-time coordinates and momenta of a $p$-brane $\left(x^{\underline{a}}, p_{\underline{a}}\right)$. The action of $p$-brane obtained from the nonlinear realization in the reduced space leads to the canonical action of a Dirac-NambuGoto $p$-brane in configuration space. The canonical Lagrangian becomes

$$
\mathcal{L}=\int d^{p} \xi\left(p_{\underline{a}} \dot{x}^{\underline{a}}-\left(\hat{\lambda}^{\perp} \mathcal{H}_{\perp}^{1}+\hat{\lambda}^{m} \mathcal{H}_{m}^{1}\right)\right),
$$

with

$$
\mathcal{H}_{\perp}^{1}=\frac{1}{2 \kappa}\left(\eta \underline{a b} p_{\underline{a}} p_{\underline{b}}+\kappa^{2} \mathbf{g}\right), \quad \mathcal{H}_{m}^{1}=p_{\underline{a}} \partial_{m} x^{\underline{a}} .
$$

In summary the action (2.8), which depends on the coordinates of the $p$-brane and the Goldstone bosons associated to the broken Lorentz rotations, leads in a natural way to the canonical action of a $p$-brane.

\section{Corrections to the NG Lagrangian}

In section 2 we have constructed in generic dimensions an invariant action for $p$-brane in terms of the MC forms of unbroken translations. We have also constructed the WZ Lagrangian (quasi invariant Lagrangian) in dimension $p+2$ for a $p$-brane using the MC forms of all translations. In this section we will see that we can use the $\mathrm{MC}$ forms of the broken Lorentz rotations to discuss possible terms of the effective action of a $p$-brane ${ }^{20}$. In order to construct these terms we will need to use the worldvolume geometry, in particular the Hodge star operator on the $\mathrm{MC}$ forms. According to the dimensionality of the couplings, the candidate Lagrangians built up with the MC forms will be added as corrections to the lowest order Lagrangian (2.8). As was mentioned in the introduction we make a perturbative dynamical determination of the variables associated with the broken Lorentz rotations to obtain higher order derivative corrections to the Nambu-Goto Lagrangian instead of the minimal use of the IH condition[12]. In the spirit of the effective theories, the coefficients of these terms will be small compared to the NG term. After considering general properties of the $p$-brane we will consider in some detail the case of the particle and the string.

\footnotetext{
${ }^{19}$ Note that in this gauge, Eq.(2.40) becomes the parametrization of Lorentz transformations used in [10].

${ }^{20}$ In [17] [18] the MC forms associated to broken Lorentz rotations were used to construct relativistic (super) particle gauged fixed action in two dimensions with extrinsic curvature.
} 


\section{$3.1 p$-brane}

First we note that the volume form for $p$-brane is expressed in terms of Hodge operator $*$ as

$$
\mu=L^{0} \ldots L^{p}=\frac{1}{p+1} L^{a} * L_{a}=d^{p+1} \xi e,
$$

where the Hodge $*$ on $q$ form is defined by $\left(L^{b_{1}} \ldots L^{b_{q}}\right) *\left(L_{a_{1}} \ldots L_{a_{q}}\right)=\mu \delta_{a_{1}}^{b_{1}} \ldots \delta_{a_{q}}^{b_{q}},\left(\right.$ for $b_{1}<$ $\left.\ldots<b_{q}, a_{1}<\ldots<a_{q}\right)$. The NG action of a $p$-brane is proportional to the volume form (3.1), see (2.9). The equations of motion for the NG $p$-brane are expressed as

$$
\delta \Phi \rightarrow L^{a^{\prime}}=0, \quad \delta x^{\underline{a}} \rightarrow L^{a b^{\prime}} * L_{a}=0 .
$$

The first one is obtained by the variation with respect to $\Phi$ and is known as the IH condition [12]. In the following we construct a geometric $p$-brane action using with the MC forms $L^{a}, L^{a b^{\prime}}$ and solve the EOM of $\Phi$ iteratively. By eliminating $\Phi$ up to a given order a geometric effective action is obtained as a function of higher derivative of the world-volume coordinates $x^{\underline{a}}$.

As possible corrections to the NG action we here consider some invariant $p+1$ forms,

$$
R^{a b} *\left(L_{a} L_{b}\right), \quad L^{a b^{\prime}} * L_{a b^{\prime}}, \quad K^{a^{\prime}} * K_{a^{\prime}}, \quad R^{a b} * R_{a b}
$$

where

$$
R^{a b}=d L^{a b}+L^{a c} L_{c}^{b}=L^{a c^{\prime}} L_{c^{\prime}}^{b},
$$

is the curvature two form and

$$
K^{a^{\prime}}=\frac{-1}{p !} \epsilon_{a_{0} a_{1} \ldots a_{p}} L^{a_{0} a^{\prime}} L^{a_{1}} \ldots L^{a_{p}}=L^{a a^{\prime}} * L_{a}
$$

is essentially the trace of the extrinsic curvature expressed as a $p+1$ form transverse vector. Explicitly

$$
\begin{aligned}
R^{a b} *\left(L_{a} L_{b}\right) & =d^{p+1} \xi e e_{a}{ }^{i} e_{b}{ }^{j]}\left(\partial_{i} \Phi_{\underline{a}}{ }^{a}\left(\eta^{\underline{a b}}-\Phi^{\underline{a}}{ }_{c} \Phi^{\underline{b} c}\right) \partial_{j} \Phi_{\underline{b}}{ }^{b}\right) \equiv d^{p+1} \xi e \mathcal{R}, \\
R^{a b} * R_{a b} & =\frac{1}{2} d^{p+1} \xi e \mathcal{R} \cdot \mathcal{R}, \quad K^{a^{\prime}}=-d^{p+1} \xi e e_{b}{ }^{i}\left(\partial_{i} \Phi^{b} \cdot \Phi^{a^{\prime}}\right) \equiv-d^{p+1} \xi e \mathcal{K}^{a^{\prime}}, \\
K^{a^{\prime}} * K_{a^{\prime}} & =d^{p+1} \xi e \mathcal{K}^{a^{\prime}} \mathcal{K}_{a^{\prime}}=d^{p+1} \xi e e_{a}{ }^{i} e_{b}{ }^{j}\left(\partial_{i} \Phi_{\underline{a}}{ }^{a}\left(\eta^{\underline{a b}}-\Phi^{\underline{a}}{ }_{c} \Phi^{\underline{b}}\right) \partial_{j} \Phi_{\underline{b}}{ }^{b}\right) \\
L^{a b^{\prime}} * L_{a b^{\prime}} & =d^{p+1} \xi e e_{a}{ }^{i} e^{a j}\left(\partial_{i} \Phi_{\underline{a}}{ }^{b}\left(\eta^{\underline{a b}}-\Phi^{\underline{a}}{ }_{c} \Phi^{\underline{b} c}\right) \partial_{j} \Phi_{\underline{b} b}\right) .
\end{aligned}
$$

More correctly $\mathcal{R}$ and $\mathcal{K}^{a^{\prime}}$ become Riemannian and extrinsic curvatures at lowest order of $\beta$ (NG $p$-brane) (see e.g. (3.60)). Note also that the first three quantities in (3.3) are not independent at the lowest order of string, but related by the GaussCodazzi equation, see for example [28]. In (3.3) we didn't consider to use the forms $L^{a^{\prime}}$ because they vanish at lowest order of the EOM for $\Phi$ (3.2). They only contribute in higher order perturbations. On the other hand we do keep the extrinsic curvatures $K^{b^{\prime}}$ which vanish when the EOM of $x \underline{a}$ of the NG brane is taken. We use the EOM of $\Phi$ but not the EOM of $x^{\underline{a}}$ in constructing the effective action. This procedure is different from [2] [3] where the extrinsic curvatures are ignored in the effective action by using the EOM of $x^{\underline{a}}$ 's at lowest order.

The mass dimension of the volume form (NG term) is $[\mu]=m^{-1-p}$ and the terms in (3.3) have dimensions

$$
\left[R^{a b} *\left(L_{a} L_{b}\right)\right]=m^{1-p}, \quad\left[L^{a b^{\prime}} * L_{a b^{\prime}}\right]=m^{1-p}, \quad\left[K^{a^{\prime}} * K_{a^{\prime}}\right]=m^{1-p}, \quad\left[R^{a b} * R_{a b}\right]=m^{3-p} .
$$


In the following we will examine the Lagrangian by adding a linear combination of these terms -we pick those that are independent at the lowest order- whose coefficients are scaled by the brane tension $\kappa,\left([\kappa]=m^{p+1}\right)$,

$$
\mathcal{L}=-\kappa^{\prime} e+\frac{\beta_{1}}{\kappa^{\frac{1-p}{1+p}}} e \mathcal{R}+\frac{\beta_{3}}{2 \kappa^{\frac{1-p}{1+p}}} e \mathcal{K}^{a^{\prime}} \mathcal{K}_{a^{\prime}}+\frac{\beta_{2}}{\kappa^{\frac{3-p}{1+p}}} e \mathcal{R} \cdot \mathcal{R}
$$

where the Lagrange multiplier terms are included in $\kappa^{\prime}(2.13)^{21}$ and the constants $\beta$ 's are dimensionless.

\subsection{Particle}

In case of relativistic bosonic particle $(p=0)$ the NG particle action is proportional to the invariant one form $L^{0}$. We define the Hodge operator on the world-line by, $\left(\tau=\xi^{0}\right)$

$$
\left(L^{0}\right)^{*} *\left(L_{0}\right)^{*}=d \tau e=\left(L^{0}\right)^{*}
$$

which implies

$$
* d \tau=-\frac{1}{e}=-\frac{1}{\left(\dot{x}^{\underline{a}} \Phi_{\underline{a}}^{0}\right)} .
$$

Since there is no two form $R^{a b}$ on the world-line we only consider the $\mathcal{K}^{2}$ term in the action (3.8). Using $K^{a^{\prime}}=L^{0 a^{\prime}}$,

$$
K^{a^{\prime}} * K_{a^{\prime}}=L^{0 a^{\prime}} * L_{a^{\prime}}^{0}=\left(d \tau \Phi_{\underline{b} 0} \dot{\Phi}^{\underline{b}} a^{\prime}\right)\left(-\frac{\left(\Phi_{\underline{a}}{ }^{0} \dot{\Phi} \underline{a} a^{\prime}\right)}{e}\right)=-\frac{d \tau}{e} \dot{\Phi}^{a}{ }_{0}\left(\eta_{\underline{a b}}-\Phi_{\underline{a} 0} \Phi_{\underline{b}}{ }^{0}\right) \dot{\Phi}^{\underline{b} 0}
$$

Eq.(3.8) becomes

$$
\mathcal{L}=-m\left\{e+\frac{\gamma}{2}\left(\Phi_{\underline{a}}^{0} \Phi^{\underline{a} 0}+1\right)+\frac{\beta}{2 m^{2} e} \dot{\Phi}^{\underline{a}}{ }_{0}\left(\eta_{\underline{a b}}-\Phi_{\underline{a} 0} \Phi_{\underline{b}}{ }^{0}\right) \dot{\Phi}^{\underline{b} 0}\right\}
$$

where $\beta$ is a dimensionless constant and $[m]=m^{1}$. Redefining $\gamma$ in Eq.(3.12) in order to absorb $\Phi_{\underline{b}}^{0} \dot{\Phi}^{\underline{b} 0}$ terms it becomes (in units $m=1$ )

$$
\mathcal{L}=-e-\frac{\gamma}{2}\left(\Phi^{2}+1\right)-\frac{\beta}{2 e} \dot{\Phi}^{2}, \quad(\Phi)_{\underline{a}}=\Phi_{\underline{a}}{ }^{0}, \quad e=\dot{x}^{\underline{a}} \Phi_{\underline{a}}{ }^{0} .
$$

the $\beta$ term is considered as a perturbation of the free particle action ${ }^{22}$. In order to make contact with an effective Lagrangian written in terms of the geometric quantities of the world-line we solve the equations of motion of $\Phi$ perturbatively in $\beta$, in terms of the higher velocities of the particle.

The EL equations of $\Phi$ are

$$
\delta \Phi \quad ; \quad-\dot{x}^{\underline{a}}-\gamma \Phi^{\underline{a}}+\beta \frac{d}{d \tau}\left(\frac{\dot{\Phi} \underline{a}}{e}\right)+\beta \frac{\dot{\Phi}^{2}}{2 e^{2}} \dot{x}^{\underline{a}}=0,
$$

and that of $\gamma$ gives

$$
\delta \gamma \quad ; \quad \Phi^{2}+1=0
$$

\footnotetext{
${ }^{21}$ We could also have considered invariant terms with higher order derivatives.

${ }^{22} \mathrm{We}$ could have also added terms with higher derivatives of $\Phi$, like for example $\tilde{D} * L^{0 a^{\prime}} *\left(\tilde{D} * L^{0 a^{\prime}}\right)$, where $\tilde{D}$ is the covariant derivative with respect to rotations.
} 
Using the second we obtain

$$
\Phi^{\underline{a}}=\frac{1}{1+\beta \frac{(D \Phi)^{2}}{2}}\left[-D x^{\underline{a}}+\beta\left(\frac{(D \Phi)^{2}}{2} D x^{\underline{a}}+D^{2} \Phi^{\underline{a}}\right)\right],
$$

where "derivation $D$ " is defined by $D \equiv \frac{1}{e} \frac{d}{d \tau}$. Although it is a dynamical equation it is solved for $\Phi$ iteratively in $\beta$,

$\left.\Phi^{\underline{a}}=-D x^{\underline{a}}+\beta\left((D \Phi)^{2} D x^{\underline{a}}+D^{2} \Phi^{\underline{a}}\right)\right)-\beta^{2}\left(\frac{(D \Phi)^{2}}{2}\left((D \Phi)^{2} D x^{\underline{a}}+D^{2} \Phi^{\underline{a}}\right)\right)+O\left(\beta^{3}\right)$.

Using $(D x)^{2}=\frac{\dot{x}^{2}}{e^{2}}$ it follows

$$
\begin{aligned}
e^{2} & =\frac{-\dot{x}^{2}}{1-\beta^{2} J_{e}^{2}}, \\
J_{e}^{2} & \equiv \frac{\left((D \Phi)^{2}\right)^{2}}{4}\left(1+(D x)^{2}\right)+(D \Phi)^{2}\left(D^{2} \Phi \cdot D x\right)+\left(D^{2} \Phi\right)^{2} .
\end{aligned}
$$

It tells that the correction of $e$ from $\sqrt{-\dot{x}^{2}}$ starts from an $O\left(\beta^{2}\right)$ term

$$
e=\sqrt{-\dot{x}^{2}}\left(1+\frac{\beta^{2}}{2} J_{e}^{2}+O\left(\beta^{4}\right)\right)
$$

This result will be used to rewrite the derivative $D$ in terms of proper time derivative $\frac{d}{d s} \equiv D_{s}=\frac{1}{\sqrt{-\dot{x}^{2}}} \frac{d}{d \tau}$ plus corrections. Remember $\left(D_{s} x\right)^{2}=-1$.

The effective action is obtained by using (3.20) and (3.17) in the original action (3.13),

$$
\mathcal{L}=-e\left(1+\frac{\beta}{2}(D \Phi)^{2}\right)
$$

The expression of $\Phi^{\underline{a}}$ up to order $\beta^{2}$ is given by

$$
\begin{aligned}
& \Phi^{\underline{a}}=-U^{\underline{a}}-\beta \Sigma^{\underline{a}}+\beta^{2}\left(-C^{\underline{a}}+\frac{A^{2}}{2} \Sigma^{\underline{a}}-\frac{(A \Sigma)}{2} A^{\underline{a}}-\frac{\Sigma^{2}}{2} U^{\underline{a}}\right)+O\left(\beta^{3}\right), \\
& U^{\underline{a}}=D_{s} x^{\underline{a}} \\
& A^{\underline{a}}=D_{s} U^{\underline{a}}=D_{s}^{2} x^{\underline{a}} \\
& \Sigma^{\underline{a}}=D_{s} A^{\underline{b}}\left(\delta_{\underline{b}} \underline{a}-\frac{D_{s} x_{\underline{b}} D_{s} x^{\underline{a}}}{\left(D_{s} x D_{s} x\right)}\right)=D_{s}^{3} x^{\underline{a}}-\left(D_{s}^{2} x D_{s}^{2} x\right) D_{s} x^{\underline{a}}, \\
& \Xi^{\underline{a}}=D_{s} \Sigma^{\underline{b}}\left(\delta_{\underline{b}} \underline{a}-\frac{D_{s} x_{\underline{b}} D_{s} x^{\underline{a}}}{\left(D_{s} x D_{s} x\right)}\right)=D_{s}^{4} x^{\underline{a}}-\left(D_{s}^{2} x D_{s}^{2} x\right) D_{s}^{2} x^{\underline{a}}-3\left(D_{s}^{2} x D_{s}^{3} x\right) D_{s} x^{\underline{a}}, \\
& C^{\underline{a}}=D_{s} \Xi^{\underline{b}}\left(\delta_{\underline{b}} \underline{a}-\frac{D_{s} x_{\underline{b}} D_{s} x^{\underline{a}}}{\left(D_{s} x D_{s} x\right)}\right)=D_{s}^{5} x^{\underline{a}}-\left(D_{s}^{2} x D_{s}^{2} x\right) D_{s}^{3} x^{\underline{a}}-5\left(D_{s}^{2} x D_{s}^{3} x\right) D_{s}^{2} x^{\underline{a}} \\
& \quad-\left\{3\left(D_{s}^{3} x D_{s}^{3} x\right)+4\left(D_{s}^{2} x D_{s}^{4} x\right)-\left(D_{s}^{2} x D_{s}^{2} x\right)^{2}\right\} D_{s} x^{\underline{a}} .
\end{aligned}
$$

where $U^{\underline{a}}$ is the velocity, we have also the space-like vectors acceleration, $A^{\underline{a}}$, relativistic jerk, $\Sigma^{\underline{a}}$, snap $\Xi^{\underline{a}}$, crackle $C^{\underline{a}}[19]$ [20]. The following relations are verified by 
theses vectors

$$
\begin{aligned}
U^{2} & =\left(D_{s} x D_{s} x\right)=-1 \\
A^{2} & =\left(D_{s}^{2} x D_{s}^{2} x\right)=\kappa_{1}^{2} \\
(A \Sigma) & =\left(D_{s}^{2} x D_{s}^{3} x\right)=\frac{1}{2} \kappa_{1} D_{s} \kappa_{1}, \\
\Sigma^{2} & =\left(D_{s}^{3} x D_{s}^{3} x\right)+\left(D_{s}^{2} x D_{s}^{2} x\right)^{2}=\left(\left(D_{s} \kappa_{1}\right)^{2}+\kappa_{1}^{2} \kappa_{2}^{2}\right),
\end{aligned}
$$

where $\kappa^{1}, \kappa^{2}$ are the first and second curvature of the worldline. The Lagrangian up to order $\beta^{3}$ is

$$
\mathcal{L}=-\sqrt{-\dot{x}^{2}}\left(1+\frac{\beta}{2} L^{(1)}+\frac{\beta^{2}}{2} L^{(2)}+\frac{\beta^{3}}{2} L^{(3)}\right)+O\left(\beta^{4}\right) .
$$

Here

$$
\begin{aligned}
L^{(1)} & =\left.(D \Phi)^{2}\right|_{0}=\left(D_{s}^{2} x\right)^{2}=A^{2}=\kappa_{1}^{2} \\
L^{(2)} & =-\left(\left(\left(D_{s}{ }^{2} x\right)^{2}\right)^{2}+\left(D_{s}{ }^{3} x\right)^{2}\right)=-\Sigma^{2}=-\left(\kappa_{1}^{2} \kappa_{2}^{2}+\left(D_{s} \kappa_{1}\right)^{2}\right) \\
L^{(3)} & =\frac{3}{2}\left(\left(D_{s}{ }^{2} x\right)^{2}\right)^{3}+\frac{5}{2}\left(D_{s}{ }^{2} x\right)^{2}\left(D_{s}{ }^{3} x\right)^{2}+12\left(D_{s}^{3} x D_{s}^{2} x\right)^{2}+\left(D_{s}{ }^{4} x\right)^{2} \\
& =\Xi^{2}+\frac{1}{2} A^{2} \Sigma^{2}-(A \Sigma)^{2} .
\end{aligned}
$$

If we use another seed Lagrangian, for instance by introducing a non-polynomial term,

$$
\mathcal{L}=-e\left(1+\frac{\tilde{\beta}}{2} \sqrt{(D \Phi)^{2}}\right),
$$

(Note that the last term of (3.30) is obtained from the MC forms in the worldline by computing the square root of a scalar: $L^{0} \wedge\left(\sqrt{*\left(L^{0 a^{\prime}} * L^{0 a^{\prime}}\right)}\right)=d \tau \sqrt{\dot{\Phi}^{2}}$. $)$ then the expression for $\Phi^{\underline{a}}$ will change to

$$
\Phi^{\underline{a}}=U^{\underline{a}}+\frac{\tilde{\beta}}{2 \sqrt{-\dot{x}^{2}}} \frac{A^{\underline{a}}}{\sqrt{(A)^{2}}}+\ldots,
$$

and, accordingly, the Lagrangian in terms of the embedding coordinates will also change. Up to lowest order in $\tilde{\beta}$ it becomes,

$$
\mathcal{L}=-\sqrt{-\dot{x}^{2}}\left(1+\frac{\tilde{\beta}}{2} \sqrt{\kappa_{1}^{2}}\right)
$$

Notice that the first correction in both cases, polynomial and non-polynomial, is a function of the first curvature of the world line. This result is general and it also holds when we consider the alternative procedure, already mentioned in the introduction, of constructing Diff invariant actions through the IH mechanism (1.6).

\subsection{Particle in three dimensions}

In the particular case of three dimensions we can construct a pseudo invariant Lagrangian from a $H$ invariant two form $L^{01} \wedge L^{02}$ constructed from the MC forms of broken Lorentz rotations, we have

$$
L^{01} \wedge L^{02}=d L^{12}, \quad L^{12}=-d \tau\left(\dot{\Phi}^{\underline{a} 1} \Phi_{\underline{a}}^{2}\right),
$$


therefore we can consider an action of a particle in three dimensions

$$
I=-m \int d \tau\left\{e-\frac{\gamma_{\underline{c d}}}{2}\left(\eta^{\underline{a b}} \Phi_{\underline{a}}^{\underline{c}} \Phi_{\underline{b}} \underline{\underline{d}}-\eta \underline{\underline{c d}}\right)+\frac{\beta}{m}\left(\dot{\Phi}^{\underline{a} 1} \Phi_{\underline{a}}{ }^{2}\right)\right\} .
$$

The EOM of $\gamma$ gives the orthonormality of $\Phi$ 's and that of $\Phi$ gives

$$
\dot{x}^{\underline{a}}-\gamma_{0 \underline{c}} \Phi^{\underline{a c}}=0, \quad \gamma_{b^{\prime} \underline{c}}+\epsilon_{b^{\prime} d^{\prime}} \frac{\beta}{m} \dot{\Phi}^{\underline{a} d^{\prime}} \Phi_{\underline{a c}}=0 .
$$

Using the second one the first equation becomes

$$
\dot{x}^{\underline{a}}-\gamma_{00} \Phi^{\underline{a} 0}-\frac{\beta}{m} \Phi_{\underline{b} 0} \epsilon_{c^{\prime} d^{\prime}} \dot{\Phi}^{\underline{b} c^{\prime}} \Phi^{\underline{a} d^{\prime}}=0 .
$$

Saturating this last equation with $\Phi_{\underline{a}}{ }^{0}$ we get $e=-\gamma_{00}$ and

$$
\Phi^{\underline{a} 0}=-\frac{1}{e}\left(\dot{x}^{\underline{a}}-\frac{\beta}{m} \Phi_{\underline{b} 0} \epsilon_{c^{\prime} d^{\prime}} \dot{\Phi^{b} c^{\prime}} \Phi^{\underline{a} d^{\prime}}\right) .
$$

On the other hand, saturating Eq.(3.36) with $\Phi_{\underline{a}}{ }^{b^{\prime}}$, we obtain

$$
\dot{x}^{\underline{a}} \Phi_{\underline{a}}{ }^{b^{\prime}}=\frac{\beta}{m} \Phi_{\underline{b} 0} \dot{\Phi}^{\underline{b}} c^{\prime} \epsilon^{c^{\prime} b^{\prime}}
$$

which is the correction of the IH condition (1.7). Saturating Eq.(3.37) with $\dot{x}_{\underline{a}}$ we can express $e$ as

$$
e=\sqrt{-\dot{x}^{2}}\left(1+\frac{\beta^{2}}{2 m^{2}\left(-\dot{x}^{2}\right)}\left(\Phi_{\underline{a} 0} \dot{\Phi}^{a} b^{\prime}\right)^{2}\right) .
$$

Using it and Eq.(3.37) we can rewrite the Lagrangian (3.34). In doing so, since $S O(2)$ is a symmetry of Eq.(3.34), one can make different choices for $\Phi^{\underline{a}}{ }^{1}, \Phi^{\underline{a}} 2$, compatible with Eq.(3.37). Each choice amounts to a complete gauge fixing of the $S O(2)$ invariance. A possible choice is

$$
\begin{aligned}
& \Phi^{\underline{a} 0}=-D_{s} x^{\underline{a}}-\frac{\beta}{m}\left(\left(D_{s} \Phi_{\underline{b}}{ }^{0}\right) \Phi^{\underline{b}}{ }_{c^{\prime}} \epsilon^{c^{\prime} d^{\prime}}\right) \Phi^{\underline{a}}{ }_{d^{\prime}}, \\
& \Phi^{\underline{a} 1}=\frac{D_{s}^{2} x^{\underline{a}}}{\sqrt{\left(D_{s}^{2} x\right)^{2}}}-\frac{\beta}{m}\left(\left(D_{s} \Phi_{\underline{b}}{ }^{0}\right) \Phi^{\underline{b}}{ }_{c^{\prime}} \epsilon^{c^{\prime} 1}\right) \Phi^{\underline{a} 0}, \\
& \Phi^{\underline{a} 2}=\frac{\epsilon^{\underline{a b c}}\left(D_{s} x_{\underline{b}}\right)\left(D_{s}^{2} x_{\underline{c}}\right)}{\sqrt{\left(D_{s}^{2} x\right)^{2}}}-\frac{\beta}{m}\left(\left(D_{s} \Phi_{\underline{b}}{ }^{0}\right) \Phi^{\underline{b}} c^{\prime} \epsilon^{c^{\prime} 2}\right) \Phi^{\underline{a} 0} .
\end{aligned}
$$

The action (3.34) becomes, up to $O\left(\beta^{2}\right)$

$$
\left.I=-m \int d s\left(1+\left.\frac{\beta}{m}\left(D_{s} \Phi^{\underline{a} 1} \Phi_{\underline{a}}^{2}\right)\right|_{N G}+\left.\frac{\beta^{2}}{m^{2}}\left(D_{s} \Phi_{\underline{a}}{ }^{0} \Phi^{\underline{a} b^{\prime}}\right)^{2}\right|_{N G}\right)\right)+O\left(\beta^{3}\right),
$$

where $\beta^{2}$ term comes from both $e$ and $\frac{\beta}{m}\left(\dot{\Phi}^{\underline{a}}{ }^{1} \Phi_{\underline{a}}{ }^{2}\right)$ terms in $(3.34) .{ }^{23}$ Here $\left.A\right|_{N G}$ means $A$ is evaluated at $O\left(\beta^{0}\right)$. It is written as

$$
I=-m \int d s\left(1+\frac{\beta}{m} \kappa_{2}+\frac{\beta^{2}}{m^{2}} \kappa_{1}^{2}\right)+O\left(\beta^{3}\right) .
$$

\footnotetext{
${ }^{23}$ Different parametrizations of $\Phi$ 's connected by local $\mathrm{SO}(2)$ give equivalent action since $\left.\left(D_{s} \Phi^{\underline{a} 1} \Phi_{\underline{a}}{ }^{2}\right)\right|_{N G}$ differs by a surface term and $\left.\left(D_{s} \Phi_{\underline{a}}{ }^{0} \Phi^{\underline{a} b^{\prime}}\right)^{2}\right|_{N G}$ is $\mathrm{SO}(2)$ invariant.
} 
The $O(\beta)$ term $\kappa_{2}$ is the torsion and $\kappa_{1}$ in $O\left(\beta^{2}\right)$ term is the curvature, all defined in (3.27)-(3.28). Particle dynamics with the torsion term has been discussed in [39] and in non-covariant gauge -corresponding to another choice instead of $(3.41),(3.41)$ in [40] [41]. Both Lagrangians differ by a total derivative.

\subsection{String}

The NG action for the string is constructed from the $S O(1,1) \times S O(D-2)$ invariant two form $\frac{1}{2} \epsilon_{a b} L^{a} \wedge L^{b}=L^{0} \wedge L^{1} \equiv \mu$, which is the volume form of the world-sheet (2.9). The Hodge operator for one form is

$$
L_{a} \wedge * L^{b}=\delta_{a}{ }^{b} \mu, \quad \mu=d^{2} \xi \operatorname{det} e
$$

then the Hodge operations on $L^{a}$ and $d \xi^{i}$ are

$$
* L_{a}=-\epsilon_{a b} L^{b}, \quad * d \xi^{i}=-e g^{i j} \epsilon_{j k} d \xi^{k},
$$

where $g^{i j}$ is the inverse of the metric of the world-sheet $g_{i j}=e_{i}{ }^{a} e_{i}{ }^{b} \eta_{a b}$ and $e=$ $\operatorname{det} e=-\frac{1}{2}\left(\epsilon_{a b} \epsilon^{i j} e_{i}{ }^{a} e_{j}{ }^{b}\right)$. Using (3.6) we can write the invariant scalar local density (3.8) for the string as

$$
\mathcal{L}=-\kappa^{\prime} e+\frac{\beta_{1}}{\kappa} e \mathcal{R}+\frac{\beta_{3}}{2} e \mathcal{K}^{2}+\frac{\beta_{2}}{\kappa} e \mathcal{R}^{2} .
$$

The dimension of the string tension is $[\kappa]=m^{2}$ and the $\beta$ 's are dimensionless. The Lagrangian is described by $x^{\underline{a}}, \Phi_{\underline{a}} \underline{b}$ and the Lagrange multipliers $\gamma_{\underline{c} d}$. The first term is the NG Lagrangian and the second term is a total divergence. If we ignore the surface term Eq.(3.47) becomes

$$
\begin{aligned}
\mathcal{L}= & -\kappa e+\frac{e}{2} \gamma_{\underline{c} \underline{d}}\left(\Phi_{\underline{a}}{ }^{\underline{c}} \Phi_{\underline{b}}{ }^{\underline{d}} \eta^{\underline{a b}}-\eta \underline{c d}\right)+\frac{\beta_{2}}{\kappa e}\left(\epsilon_{a b} \epsilon^{i j} \partial_{i} \Phi_{\underline{a}}{ }^{a} O^{\frac{a b}{\Phi}} \partial_{j} \Phi_{\underline{b}}{ }^{b}\right)^{2} \\
& +\frac{\beta_{3}}{2} e\left(e_{a}{ }^{i} \partial_{i} \Phi_{\underline{a}}{ }^{a}\right) O O_{\Phi} \underline{a b}\left(e_{b}{ }^{j} \partial_{j} \Phi_{\underline{b}}{ }^{b}\right) \equiv-\kappa^{\prime} e+\beta \mathcal{L}^{\prime} .
\end{aligned}
$$

where $O \frac{a b}{\Phi}=\left(\eta \underline{a b}-\Phi^{\underline{a}}{ }^{\underline{a}} \Phi_{c}\right)$. For small $\beta$ 's we may solve the EOM for $\Phi$ perturbatively and the resulting geometrical Lagrangian depends on higher order derivatives of $x$. The EOM of $\Phi$ is

$$
-\kappa e e_{b}{ }^{i} \partial_{i} x^{\underline{a}} \delta_{\underline{b}}^{b}+e \gamma_{\underline{b d}} \Phi^{\underline{a d}}+\beta\left(\mathcal{L}^{\prime}\right)^{\underline{a}}{ }_{\underline{b}}=0, \quad\left(\mathcal{L}^{\prime}\right)^{\underline{a}} \underline{\underline{b}} \equiv \frac{\delta \mathcal{L}^{\prime}}{\delta \Phi_{\underline{a}} \underline{b}} .
$$

Since $\mathcal{L}^{\prime}$ does not depends on $\Phi_{\underline{a}}{ }^{b^{\prime}},\left(\mathcal{L}^{\prime}\right) \underline{a}_{b^{\prime}}=0$. Taking $\underline{b}=b^{\prime}$ in Eq.(3.49) we obtain

$$
\gamma_{b^{\prime} d}=\gamma_{b^{\prime} d^{\prime}}=0
$$

whereas from the $\underline{b}=b$ component of Eq.(3.49) we can determine

$$
\gamma_{a b}=\kappa \eta_{a b}-\frac{\beta}{e}\left(\mathcal{L}^{\prime}\right)^{\underline{a}}{ }_{a} \Phi_{\underline{a} b}, \quad\left(\mathcal{L}^{\prime}\right)^{\underline{a}}{ }_{[a} \Phi_{\underline{a} b]}=0 .
$$

The latter equality holds identically due to the local $S O(1,1)$ invariance of $\mathcal{L}^{\prime}$ under $\delta \Phi_{\underline{a}}{ }^{b}=\alpha^{b}{ }_{c} \Phi_{\underline{a}}^{c},\left(\alpha^{b c}+\alpha^{c b}=0\right)$. Plugging (3.50) and (3.51) into (3.49),

$$
\Phi^{\underline{a}}{ }_{b}=e_{b}^{i} \partial_{i} x^{\underline{a}}-\frac{\beta}{\kappa e} O_{\Phi}^{\underline{a b}}\left(\mathcal{L}^{\prime}\right)_{\underline{b} b} .
$$


Now, saturating Eq.(3.52) with $\Phi_{\underline{a}}{ }^{c^{\prime}}$ and using the orthogonality relations we get

$$
\partial_{i} x^{\underline{a}} \Phi_{\underline{a}}{ }^{\prime}=\frac{\beta}{\kappa e} e_{i}^{b}\left(\mathcal{L}^{\prime}\right) \underline{a}{ }_{b} \Phi_{\underline{a}}{ }^{\prime}
$$

which is the correction of the IH condition. Multiplying $\partial_{i} x_{\underline{a}}$ on (3.52)

$$
e_{i}^{b}=\partial_{i} x^{\underline{a}} \Phi_{\underline{a}}{ }^{b}=\eta^{b c} e_{c}^{j} \bar{g}_{j i}-\left(\frac{\beta}{\kappa e}\right)^{2} e_{i}^{b}\left(\mathcal{L}^{\prime}\right)_{\underline{a} c} O_{\Phi}^{\underline{a b}}\left(\mathcal{L}^{\prime}\right)_{\underline{b}}{ }^{c},
$$

where $\bar{g}_{j i}$ is the induced metric,

$$
\bar{g}_{j i}=\left(\partial_{j} x_{\underline{a}} \partial_{i} x^{\underline{a}}\right) .
$$

Taking its determinant we get an expansion of $e$

$$
e=\operatorname{det} e=\sqrt{-\bar{g}}\left(1-\frac{1}{2}\left(\frac{\beta}{\kappa e}\right)^{2}\left(\mathcal{L}^{\prime}\right)_{\underline{a} b} O_{\bar{\Phi}}^{\underline{a b}}\left(\mathcal{L}^{\prime}\right)_{\underline{b}}^{b}\right)+O\left(\beta^{3}\right) .
$$

We use (3.52) and (3.56) to rewrite the Lagrangian (3.48) up to $O\left(\beta^{2}\right)$ as

$$
\begin{aligned}
\mathcal{L} & =-\kappa \sqrt{-\bar{g}}\left(1+\left.\beta \mathcal{L}^{\prime}\right|_{N G}+\left.\left(\frac{1}{2}\left(\frac{\beta}{\kappa e}\right)^{2}\left(\mathcal{L}^{\prime}\right)_{\underline{a} b} O \frac{a b}{\Phi}\left(\mathcal{L}^{\prime}\right)_{\underline{b}}{ }^{b}\right)\right|_{N G}\right) \\
& =-\kappa \sqrt{-\bar{g}}\left(1+\left.\frac{\beta_{2}}{\kappa} \mathcal{R}^{2}\right|_{N G}+\left.\frac{\beta_{3}}{2} \mathcal{K}^{2}\right|_{N G}+\left.\left(\frac{1}{2}\left(\frac{\beta}{\kappa e}\right)^{2}\left(\mathcal{L}^{\prime}\right)_{\underline{a} b} O_{\Phi}^{\underline{a b}}\left(\mathcal{L}^{\prime}\right)_{\underline{b}}{ }^{b}\right)\right|_{N G}\right) .
\end{aligned}
$$

Here $\left.A\right|_{N G}$ means $A$ is evaluated at $O\left(\beta^{0}\right)$ order, where the IH condition $\partial_{i} x^{\underline{a}} \Phi_{\underline{a}}{ }^{a^{\prime}}=0$ holds and $\Phi_{\underline{a}}{ }^{a}$ 's are tangential to the world-sheet.

Up to $S \bar{O}(1,1)$ gauge freedom we can choose, for example,

$$
\Phi^{\underline{a} 0}=-\frac{\dot{x}^{\underline{a}}}{\sqrt{-\dot{x}^{2}}}, \quad \Phi^{\underline{a} 1}=\frac{x^{\prime} \underline{\underline{a}}}{\sqrt{\left(x_{\perp}^{\prime}\right)^{2}}}, \quad x^{\prime} \underline{\underline{a}}=x^{\prime} \underline{a}-\frac{x^{\prime} \cdot \dot{x}}{\dot{x}^{2}} \dot{x}^{\underline{a}} .
$$

The $\left.\mathcal{R}\right|_{N G}$ is the scalar curvature of the world-sheet

$$
\left.\mathcal{R}\right|_{N G}=\frac{1}{(-\bar{g})} \epsilon^{i_{1} i_{2}} \epsilon^{j_{1} j_{2}}\left(\partial_{i_{1}} \partial_{j_{1}} x^{\underline{a}}\right)\left(\eta_{\underline{a b}}-\bar{g}^{i_{3} j_{3}} \partial_{i_{3}} x_{\underline{a}} \partial_{j_{3}} x_{\underline{b}}\right)\left(\partial_{i_{2}} \partial_{j_{2}} x^{\underline{b}}\right)
$$

and the $\mathcal{K}^{c^{\prime}}=\left.\mathcal{K}^{c^{\prime}}\right|_{N G}$ is the extrinsic curvature written as

$$
\left.\mathcal{K}^{c^{\prime}}\right|_{N G}=\left(\square x^{\underline{a}}\right) n_{\underline{a}}{ }^{c^{\prime}},
$$

here $\square$ is the $\bar{g}_{i j}$ covariant d'Alembertian and $n_{\underline{a}}{ }^{\prime}=\left.\Phi \underline{a}^{\prime}\right|_{N G}$ are normal unit vectors of the world-sheet. The $O\left(\beta^{2}\right)$ term of the Lagrangian in (3.57) should give higher order curvatures of the world-sheet. 


\section{Conclusions}

In this paper we have constructed terms of the effective action of a tensionfull $p$ brane using the non-linear realization method. We have considered the Goldstone bosons associated to the broken and unbroken translations, coordinates of the $p$ brane, and the Goldstone bosons associated to the broken Lorentz rotations. In order to avoid using an explicit parametrization of the Lorentz transformations we have introduced also Goldstone bosons associated to the unbroken rotations $S O(1, p) \times$ $S O(D-(p+1))$. The Goldstone bosons of unbroken generators could be eliminated using the corresponding gauge transformations of local rotations.

We have seen using the action with the lowest order of derivatives that the Goldstone bosons of the broken Lorentz rotations are non dynamical and can be expressed as functions of the coordinates and momenta of the $p$-brane. We have also seen how the unperturbed action leads in a natural way to the canonical action of the NG

$p$-brane. In other words the action obtained through the non-linear realizations of space-time symmetries is an action of phase space type, this result generalizes analogous results for the particle [15] [16] to the $p$-brane.

We have constructed the lower order corrections up to velocities in $x$ and $\Phi$ to the NG action. The correction terms in the spirit of the effective action are small. We do not use the IH constraint[12], instead we solve perturbatively the equations of motion for the Lorentz variables $\Phi$ which, upon substitution in the original Lagrangian, give higher order geometrical terms for the effective action. For the case of the particle and string, the actions obtained in this way are written in terms of natural geometrical objects.

Our method does not have the completeness of the IH method, which gives any possible correction terms for the NG Lagrangian of a $p$-brane. However we want to remark that it naturally selects some specific terms within the wider set of potential correction terms. It appears that there is a very natural, an non-trivial, geometric interpretation of the first terms obtained in our approach, which may suggest that there could be physical reasons behind the selection of the seeds that give raise to these terms. At this point we do not have a compelling argument for these choices, but we think that they have enough interest to be considered.

This method could be also useful in cases where the geometrical quantities are not well known, for example non-relativistic string theories, [32] [33] [34] [35] [36], Finsler type theories [37] and the corresponding supersymmetrization.

Acknowledgements We acknowledge comments from Roberto Casalbuoni, Paul Townsend and Toine Van Proeyen. J.G. acknowledges Toine Van Proeyen for the hospitality at KU Leuven where this work was completed. We also acknowledge partial financial support from projects FP2010-20807-C02-01, 2009SGR502 and CPAN Consolider CSD 2007-00042. 


\section{A MC equations of Poincare group. Geomet- rical Aspects}

The MC forms of the Poincare algebra (1.2) satisfy the MC equations

$$
d L^{\underline{a}}+L^{\underline{a b}} L_{\underline{b}}=0, \quad d L^{\underline{a b}}+L^{\underline{a c}} L_{\underline{\underline{c}}}^{\underline{b}}=0,
$$

which mean that the target space is torsionless and has no curvature.

We can consider the pullback to the world-volume $\Sigma$ and $L^{a *}=d \xi^{i} e_{i}^{a}$ as $p+1$ bein of $\Sigma$ and $L^{a b *}=d \xi^{i} \omega_{i}^{a b}$ as the spin connection. Using (A.1 $)^{24}$

$$
\begin{gathered}
d L^{a}+L^{a b} L_{b}+L^{a b^{\prime}} L_{b^{\prime}}=0, \quad \rightarrow \quad d e^{a}+\omega^{a}{ }_{b} e^{b}=-L^{a b^{\prime}} L_{b^{\prime}} \equiv T^{a}, \\
d L^{a b}+L^{a c} L_{c}{ }^{b}+L^{a c^{\prime}} L_{c^{\prime}}{ }^{b}=0 \quad \rightarrow \quad d \omega^{a b}+\omega^{a c} \omega_{c}{ }^{b}=-L^{a c^{\prime}} L_{c^{\prime}}{ }^{b} \equiv R^{a b} .
\end{gathered}
$$

Here $T^{a}$ is torsion two form of $\Sigma$, which vanishes for the NG $p$-brane since $L_{b^{\prime}}=0$

using the EOM. $R^{a b}$ is the curvature two form, $R^{a b}=\frac{1}{2} d \xi^{i} d \xi^{j} R_{i j}{ }^{a b}$. It is related to the scalar curvature $\mathcal{R}$ as follows. In general $p+1$ dimensions $\left(\epsilon^{01 \ldots}=1=-\epsilon_{01 \ldots}\right)$

$$
\begin{aligned}
& \frac{-1}{(p-1) !} \epsilon_{a_{0} \ldots a_{p}} e^{a_{0}} \ldots e^{a_{p-2}} R^{a_{p-1} a_{p}} \\
& =\frac{-1}{2(p-1) !} d^{p+1} \xi \epsilon_{a_{0} \ldots a_{p}} \epsilon^{i_{0} \ldots i_{p}} e_{i_{0}}^{a_{0}} \ldots e_{i_{p-2}}^{a_{p-2}} R_{i_{p-1} i_{p}}^{a_{p-1} a_{p}} \\
& =\frac{1}{2} d^{p+1} \xi e e_{a_{p-1}}{ }^{\left[i_{p-1}\right.} e_{a_{p}}{ }^{\left.i_{p}\right]} R_{i_{p-1} i_{p}}{ }^{a_{p-1} a_{p}}, \quad e=\operatorname{det}\left(e_{i}{ }^{a}\right), \\
& =d^{p+1} \xi e e_{a}{ }^{i} e_{b}{ }^{j} R_{i j}{ }^{a b}=d^{p+1} \xi e \mathcal{R}, \quad \mathcal{R}=e_{a}{ }^{i} e_{b}{ }^{j} R_{i j}{ }^{a b} .
\end{aligned}
$$

In the case of the string $(p=1)$,

$$
-\epsilon_{a b} R^{a b}=-\frac{1}{2} d^{2} \xi \epsilon_{a b} \epsilon^{i j} R_{i j}^{a b}=\frac{1}{2} d^{2} \xi e e_{a}{ }^{[i} e_{b}{ }^{j]} R_{i j}{ }^{a b}=d^{2} \xi e \mathcal{R},
$$

where

$$
e_{a}{ }^{[i} e_{b}^{j]}=-\epsilon_{a b} \epsilon^{i j} \operatorname{det}\left(e_{a}^{i}\right)=-\epsilon_{a b} \epsilon^{i j} / e .
$$

In addition, in this case,

$$
R^{a b}=-L^{a c^{\prime}} L_{c^{\prime}}^{b}=d \omega^{a b}+\omega^{a c} \omega_{c}^{b}=d \omega^{a b}
$$

because $\omega^{0 c} \omega_{c}{ }^{1} \equiv 0$ for the string. Then the curvature $R^{a b}$ is an exact form and, as a consequence, the scalar density $e \mathcal{R}$ is a surface term in 2-dimensions.

\section{B Canonical formalism}

In this Appendix we present the canonical formalism of the action (2.8)

$$
\mathcal{L}=-\kappa^{\prime} e=-\kappa e+e \frac{\gamma_{\underline{c d}}}{2}\left(\eta^{\underline{a b}} \Phi_{\underline{a}} \underline{\underline{c}} \Phi_{\underline{\underline{b}}}^{\underline{d}}-\eta \eta^{\underline{c d}}\right)
$$

\footnotetext{
${ }^{24}$ All expressions below are pullbacks to the world-volume. The pullback notation, $L^{a} \rightarrow L^{a *}$, etc., is omitted for simplicity.
} 
and show how the NLR action leads in a natural way to the canonical action of the NG p-brane. We will also show that the Goldstone bosons of the broken Lorentz generators are functions of the phase space variables of the $p$-brane generalizing the result of the particle in the introduction.

The canonical momenta are ${ }^{25}$

$$
\begin{aligned}
p_{\underline{a}} & =\frac{\partial \mathcal{L}}{\partial \dot{x}^{\underline{a}}}=-\kappa^{\prime} e e_{b}{ }^{0} \frac{\partial e_{0}{ }^{b}}{\partial \dot{x}^{\underline{a}}}=-\kappa^{\prime} e e_{b}{ }^{0} \Phi_{\underline{a}}{ }^{b}, \\
\Pi_{\underline{a}} \underline{b} & =\frac{\partial \mathcal{L}}{\partial \dot{\Phi}_{\underline{a}} \underline{b}}=0, \\
p_{\underline{\gamma}}^{\underline{a}} & =\frac{\partial \mathcal{L}}{\partial \dot{\gamma}_{\underline{\underline{b}}}}=0,
\end{aligned}
$$

where $e_{b}{ }^{i}$ is the inverse vielbein $e_{b}{ }^{i} e_{i}{ }^{a}=\delta^{a}{ }_{b}$. We have $e e_{a}{ }^{i}=-\frac{1}{p !} \epsilon^{i i_{1} \ldots i_{p}} \epsilon_{a a_{1} \ldots a_{p}} e_{i_{1}}{ }^{a_{1}} \ldots e_{i_{p}}{ }^{a_{p}}$. Note that the combination $e e_{b}{ }^{0}$ does not depend on the velocities $\dot{x}^{\underline{a}}$. Therefore all the definitions of momenta (B.2)-(B.4) yield primary constraints,

$$
\begin{aligned}
\phi_{\underline{a}} & \equiv p_{\underline{a}}+\kappa^{\prime} e e_{b}^{0} \Phi_{\underline{a}}^{b}=0, \\
\phi_{\underline{a}}^{\underline{a}} & \equiv \Pi^{\underline{a}} \underline{\underline{a}}=0, \\
\phi_{\gamma}^{a b} & \equiv p^{a} \underline{b}=0 .
\end{aligned}
$$

The Hamiltonian is a sum of primary constraints,

$$
\mathcal{H}_{D}=\int d^{p} \xi \mathcal{H}, \quad \mathcal{H}=\phi_{\underline{a}} \lambda^{\underline{a}}+\phi^{\underline{a}}{ }_{\underline{b}} \Lambda_{\underline{a}} \underline{b}+\phi \frac{a b}{\gamma} \lambda_{\underline{a b}}^{\gamma},
$$

where $\lambda \underline{a}, \Lambda_{\underline{a}} \underline{b}$ and $\lambda_{a b}^{\gamma}$ are arbitrary functions of $\xi$ at this moment.

In order to compute the Hamiltonian equations of motion we introduce the Poisson brackets, ( $\xi^{0}$ is temporal and $\xi^{m}, m=1, \ldots, p$, are spatial world-volume coordinates)

$$
\begin{aligned}
& \left\{x^{\underline{a}}\left(\xi^{0}, \xi^{m}\right), p_{\underline{b}}\left(\xi^{0}, \xi^{\prime m}\right)\right\}=\delta_{\underline{b}}^{\underline{a}} \delta^{p}\left(\xi^{m}-\xi^{\prime m}\right) \\
& \left\{\gamma_{\underline{a} \underline{b}}\left(\xi^{0}, \xi^{m}\right), p \frac{c d}{\gamma}\left(\xi^{0}, \xi^{\prime m}\right)\right\}=\frac{1}{2}\left(\delta_{\underline{c}} \delta_{\underline{b}}^{\underline{d}}+\delta \delta_{\underline{a}} \delta_{\underline{b}}^{\underline{c}}\right) \delta^{p}\left(\xi^{m}-\xi^{\prime m}\right) \\
& \left\{\Phi_{\underline{a}} \underline{b}\left(\xi^{0}, \xi^{m}\right), \Pi_{\underline{c}} \underline{\underline{c}}\left(\xi^{0}, \xi^{\prime m}\right)\right\}=\delta \underline{\underline{a}} \delta_{\underline{\underline{b}}}^{\underline{b}} \delta^{p}\left(\xi^{m}-\xi^{\prime m}\right)
\end{aligned}
$$

from which we obtain Hamilton's equations for the configuration variables,

$$
\begin{aligned}
\dot{x}^{\underline{a}} & =\left\{x^{\underline{a}}, \mathcal{H}_{D}\right\}=\lambda \underline{a}, \\
\dot{\gamma}_{\underline{a} b} & =\left\{\gamma_{\underline{a b}}, \mathcal{H}_{D}\right\}=\lambda_{\underline{a} \underline{\gamma}}, \\
\dot{\Phi}_{\underline{a}} \underline{b} & =\left\{\Phi_{\underline{a}} \underline{b}, \mathcal{H}_{D}\right\}=\Lambda_{\underline{a}},
\end{aligned}
$$

and the momenta,

$$
\begin{aligned}
\dot{p}_{\underline{a}} & =\left\{p_{a}, \mathcal{H}_{D}\right\}=\partial_{m}\left(\frac{\partial}{\partial \partial_{m} x^{\underline{a}}}\left(\kappa^{\prime} e e_{b}{ }^{0} \Phi_{\underline{c}}{ }^{b} \lambda \underline{c}\right)\right)=-\partial_{m}\left(\frac{\partial}{\partial \partial_{m} x^{\underline{a}}} \mathcal{L}\right), \\
\dot{p} \frac{a b}{\gamma} & =\left\{p \frac{a b}{\gamma}, \mathcal{H}_{D}\right\}=-\frac{e}{2}\left(\eta \underline{\underline{c}} \Phi_{\underline{c}}{ }^{\underline{a}} \Phi_{\underline{d}^{\underline{b}}}-\eta \eta^{\underline{a}}\right) \\
\dot{\Pi} \underline{\underline{a}}) & =\left\{\Pi \underline{\underline{a}}_{\underline{b}}, \mathcal{H}_{D}\right\}=-\frac{\partial}{\partial \Phi_{\underline{a}} \underline{b}}\left(\kappa^{\prime} e e_{b}{ }^{0} \Phi_{\underline{a}}{ }^{b} \lambda^{\underline{a}}\right)=\frac{\partial}{\partial \Phi_{\underline{a}} \underline{b}}(\mathcal{L}) .
\end{aligned}
$$

\footnotetext{
${ }^{25}$ We consider $\xi^{0}$ as the canonical time and use "dot" as $\xi^{0}$ derivative. We often do not write the dependence on $\xi^{i}$.
} 
The relations (B.12) determine the velocities $\dot{x} \underline{a}, \dot{\Phi}_{\underline{a}}^{\underline{b}}$ and $\dot{\gamma}_{\underline{a b}}^{\gamma}$ in terms of the multipliers $\lambda^{\underline{a}}, \Lambda_{\underline{a}} \underline{b}$ and $\lambda_{\underline{a b}}^{\gamma}$ respectively. Here and hereafter the velocities $\dot{x}^{\underline{a}}, \dot{\Phi}_{\underline{a}}^{\underline{b}}$ and $\dot{\gamma}_{\underline{a} b}^{\gamma}$, when appear in the Hamiltonian analysis, are to be understood as the multipliers $\lambda^{\underline{a}}, \Lambda_{\underline{a}} \underline{b}$ and $\lambda_{\underline{a b}}^{\gamma}$.

The consistency condition of the primary constraints $\dot{\phi}=0$ reproduces the EL equations of $\gamma^{\underline{a b}}, \Phi_{\underline{a}} \underline{b}$ and $x^{\underline{a}}$,

$$
\begin{aligned}
\dot{\phi} \frac{a b}{\gamma} & =\dot{p} \underline{\gamma}=-\frac{e}{2}\left(\eta \underline{c d} \Phi_{\underline{c}}^{\underline{a}} \Phi_{\underline{d}^{\underline{b}}}-\eta \underline{a b}\right)=\left(\frac{\partial}{\partial \gamma_{\underline{a b}}} \mathcal{L}\right)=0, \\
\dot{\phi}_{\underline{a}}^{\underline{b}} & =\dot{\Pi} \underline{a}_{\underline{b}}=-\frac{\partial \mathcal{H}}{\partial \Phi_{\underline{a}}^{\underline{b}}}=\frac{\partial}{\partial \Phi_{\underline{a}}^{\underline{b}}} \mathcal{L}=0 \\
\dot{\phi}_{\underline{a}} & =\dot{p}_{\underline{a}}+\partial_{\tau}\left(\kappa^{\prime} e e_{b}{ }^{0} \Phi_{\underline{a}}{ }^{b}\right)=-\partial_{m}\left(\frac{\partial}{\partial \partial_{m} x \underline{a}} \mathcal{L}\right)-\partial_{0}\left(\frac{\partial}{\partial \dot{x} \underline{a}} \mathcal{L}\right)=0 .
\end{aligned}
$$

From Eq.(B.14) we get $\frac{D(D+1)}{2}$ secondary constraints, which are the orthonormality conditions for $\Phi_{\underline{a}} \underline{\underline{b}}$

$$
\chi^{\underline{a b}} \equiv C \stackrel{\underline{a b}}{\underline{b}}=\frac{1}{2}\left(\eta^{\underline{c d}} \Phi_{\underline{\underline{c}}}^{\underline{a}} \Phi_{\underline{d}}^{\underline{b}}-\eta^{\underline{a b}}\right)=0 .
$$

The $D^{2}$ conditions (B.15) give

$$
-\kappa^{\prime} e \delta_{\underline{b}}^{b} e_{b}^{i} \partial_{i} x^{\underline{a}}+e \gamma_{\underline{b c}} \Phi \underline{a c}=0
$$

where $\gamma_{\underline{b c}}$ is symmetric by definition. The $\underline{b}=b^{\prime}$ components of Eq.(B.18) are $(D-$ $(p+1))(p+1)+\frac{(D-(p+1))(D-p)}{2}$ secondary constraint,

$$
\chi_{b^{\prime} c}^{\gamma} \equiv \gamma_{b^{\prime} c}=0, \quad \chi_{b^{\prime} c^{\prime}}^{\gamma} \equiv \gamma_{b^{\prime} c^{\prime}}=0
$$

whereas the $\underline{b}=b$ components of Eq.(B.18)

$$
-\kappa e e_{b}^{i} \partial_{i} x^{\underline{a}}+e \gamma_{b c} \Phi^{\underline{a} c}=0
$$

imply $\frac{(p+1)(p+2)}{2}$ secondary constraints,

$$
\chi_{a b}^{\gamma} \equiv \gamma_{a b}-\kappa \eta_{a b}=0,
$$

and $(p+1)(D-(p+1))$ relations

$$
\partial_{i} x^{\underline{a}} \Phi_{\underline{a}}^{b^{\prime}}=0 .
$$

Notice that Eq.(B.22) coincides with the vanishing of the MC forms $L^{b^{\prime}}$ in (A.1), associated with broken translations. The vanishing of these forms is known as the inverse Higgs mechanism [12]. Eq.(B.22) gives $p(D-(p+1))$ secondary constraints for $i=m$;

$$
\chi_{m}{ }^{b^{\prime}} \equiv \partial_{m} x^{\underline{a}} \Phi_{\underline{a}}{ }^{b^{\prime}}=0, \quad(m=1, \ldots, p) .
$$

and $(D-(p+1))$ conditions on the multipliers $\lambda^{\underline{a}}=\dot{x}^{\underline{a}}$ for $i=0$,

$$
\tilde{\lambda}^{b^{\prime}} \equiv \lambda^{\underline{a}} \Phi_{\underline{a}}^{b^{\prime}}=0
$$


The $D$ equations (B.16) do not give secondary constraints, but only $(D-(p+1))$ relations among the arbitrary functions. Let us find them. Using the notational convention mentioned after Eq.(B.13) we have

$$
\begin{aligned}
\dot{\phi}_{\underline{a}} & =\partial_{i}\left(\kappa e_{b}{ }^{i} \Phi_{\underline{a}}{ }^{b}\right)=\partial_{i}\left(\frac{-\kappa}{p !} \epsilon^{i i_{1} \ldots i_{p}} \epsilon_{b a_{1} \ldots a_{p}} e_{i_{1}}{ }^{a_{1}} \ldots e_{i_{p}}{ }^{a_{p}} \Phi_{\underline{a}}{ }^{b}\right) \\
& =\kappa e e_{[b}{ }^{i} e_{\left.a_{1}\right]}^{i_{1}}\left(\partial_{i_{1}} x^{\underline{b}_{i}}{\partial_{\underline{b}}}^{a^{1}}\right) \Phi_{\underline{a}}{ }^{b}+\kappa e e_{b}{ }^{i} \partial_{i} \Phi_{\underline{a}}{ }^{b}
\end{aligned}
$$

where

$$
\frac{-1}{(p-1) !} \epsilon^{i i_{1} \ldots i_{p}} \epsilon_{b a_{1} \ldots a_{p}} e_{i_{2}}^{a_{2}} \ldots e_{i_{p}}^{a_{p}}=e e_{[b}^{i} e_{\left.a_{1}\right]}^{i_{1}} .
$$

Then, using Eq.(B.22) and the constraints (B.17), we obtain, for $\dot{\phi}_{\underline{a}}$,

$$
\begin{aligned}
\dot{\phi}_{\underline{a}} & =\kappa e e_{[b}{ }^{i} e_{\left.a_{1}\right]}^{i_{1}}\left(\partial_{i_{1}} x^{\underline{b}} \Phi_{\underline{b}}{ }^{d}\right)\left(\Phi^{\underline{c}} \partial_{i} \Phi_{\underline{c}}{ }^{a_{1}}\right) \Phi_{\underline{a}}{ }^{b}+\kappa e e_{b}{ }^{i} \partial_{i} \Phi_{\underline{a}}{ }^{b} \\
& =\kappa e e_{b}{ }^{i} \partial_{i} \Phi_{\underline{c}}{ }^{b} \Phi^{\underline{c}} a^{\prime} \Phi_{\underline{a}}{ }^{a^{\prime}}=0,
\end{aligned}
$$

showing that only $D-(p+1)$ components of $\dot{\phi}_{\underline{a}}$, which can be conveniently taken as $\dot{\phi}_{\underline{a}} \Phi \underline{a}_{a^{\prime}}$, give independent conditions,

$$
\left(e e_{b}^{0} \Lambda_{\underline{c}}^{b}+e e_{b}^{m} \partial_{m} \Phi_{\underline{c}}^{b}\right) \Phi^{\underline{c}} a^{\prime}=0,
$$

where $e e_{b}{ }^{m},(m=1, \ldots, p)$ are linear functions of $\lambda^{\underline{a}}=\dot{x}^{\underline{a}}$.

We should further examine the stability of the secondary constraints. Let us first consider the conditions associated with the constraints $\chi^{\underline{a b}}$ in (B.17),

$$
\dot{\chi}^{\underline{a b}}=\frac{1}{2}\left(\eta^{\underline{c d}} \Lambda_{\underline{\underline{c}}}^{\underline{a}} \Phi_{\underline{d}}^{\underline{b}}+\eta^{\underline{c d}} \Phi_{\underline{\underline{c}}}^{\underline{a}} \Lambda_{\underline{\underline{d}}}^{\underline{b}}\right)=0
$$

If we redefine the arbitrary functions $\Lambda_{\underline{a}}^{\underline{b}}$ in terms of $\Omega_{\underline{a}} \underline{b}$ by

$$
\Lambda_{\underline{a}}^{\underline{b}} \equiv \Phi_{\underline{a}}^{\underline{c}} \Omega_{\underline{\underline{c}}}^{\underline{b}}
$$

then the consistency condition (B.29) imposes antisymmetry of $\Omega \underline{a b}$,

$$
\dot{\chi}^{\underline{a b}}=\frac{1}{2}(\Omega \underline{a b}+\Omega \underline{b a})=0 .
$$

On the other hand, the consistency condition of $\chi_{\underline{a b}}^{\gamma}=0$ determines the $\frac{D(D+1)}{2}$ multipliers $\lambda_{\underline{a b}}^{\gamma}$,

$$
\dot{\chi}_{\underline{a b}}^{\gamma}=\lambda_{\underline{a b}}^{\gamma}=0 .
$$

Finally, the consistency of $\chi_{m}{ }^{b^{\prime}}=0$ in (B.23) is

$$
\partial_{m} x^{\underline{a}} \Lambda_{\underline{a}}^{b^{\prime}}+\partial_{m} \lambda^{\underline{a}} \Phi_{\underline{a}}^{b^{\prime}}=e_{m}{ }^{b} \Omega_{b}{ }^{b^{\prime}}-\lambda \underline{a} \partial_{m} \Phi^{{ }^{b^{\prime}}}=0,
$$

which are $p(R-p-1)$ linear relations among multipliers $\Omega_{b}^{b^{\prime}}$ and $\lambda^{\underline{a}}$.

This finishes the analysis of constraints. No tertiary constraints arise because the dynamical consequences of the secondary constraints boil down to the partial determination of the arbitrary multipliers. This is a consistent dynamical system. 
Let us examine for further use this partial determination of the arbitrary multipliers. Combining (B.33) with (B.28), we obtain $(p+1)(D-(p+1))$ equations

$$
e e_{b}{ }^{0} \Omega_{a^{\prime}}{ }^{b}+e e_{b}{ }^{m} \partial_{m} \Phi_{\underline{c}}{ }^{b} \Phi^{\underline{c}} a^{\prime}=0, \quad e_{m}{ }^{b} \Omega_{b}{ }^{b^{\prime}}-\lambda^{\underline{a}} \partial_{m} \Phi_{\underline{a}}^{b^{\prime}}=0
$$

that can be solved for $(p+1)(D-(p+1))$ components $\Omega_{b a^{\prime}}=-\Omega_{a^{\prime} b}$ in terms of $\lambda^{\underline{a}}$. To do it we use the fact that

$$
\left(e e_{a}^{0}, e_{m}^{a}\right), \quad(a=0,1, \ldots, p)
$$

is a complete set basis in terms of canonical variables, that is, for any $A_{a}$ we can make the decomposition

$$
A_{a}=\left(e e_{a}^{0}\right) \tilde{a}_{0}+e_{m a} \tilde{a}^{m}, \quad \Leftrightarrow \quad \tilde{a}_{0}=e e_{a}{ }^{0} \eta^{a b} A_{b} /\left(e^{2} g^{00}\right), \quad \tilde{a}^{m}=\mathbf{g}^{m \ell} e_{\ell}^{b} A_{b},
$$

where $\mathbf{g}^{m \ell}$ is the inverse of the $p \times p$ matrix of $g_{m \ell}=e_{m}{ }^{a} e_{\ell}^{b} \eta_{a b}, g^{00}=e_{a}{ }^{0} e_{b}{ }^{0} \eta^{a b}=$ $\frac{\operatorname{det} g_{\ell m}}{g}=\frac{\mathbf{g}}{-e^{2}}, g=\operatorname{det} g_{i j}$ and $\mathbf{g}=\operatorname{det} g_{m \ell}$. It follows the completeness relation

$$
\delta_{a}{ }^{b}=-\frac{\left(e e_{a}^{0}\right)\left(e e^{b 0}\right)}{\mathbf{g}}+e_{m a} \mathbf{g}^{m \ell} e_{\ell}^{b}
$$

Using it $\Omega_{a^{\prime}}{ }^{b}$ is expressed in terms of $\lambda^{\underline{a}}$ as

$$
\begin{aligned}
\Omega_{a^{\prime}}^{b} & =\Omega_{a^{\prime}}^{a}\left(-\frac{\left(e e_{a}^{0}\right)\left(e e^{b 0}\right)}{\mathbf{g}}+e_{m a} \mathbf{g}^{m \ell} e_{\ell}^{b}\right) \\
& =\left(e e_{d}{ }^{m} \partial_{m} \Phi_{\underline{c}}{ }^{b} \Phi^{\underline{c}} a^{\prime}\right) \frac{\left(e e^{b 0}\right)}{\mathbf{g}}+\left(-\lambda{ }^{\underline{a}} \partial_{m} \Phi_{\underline{a} a^{\prime}}\right) \mathbf{g}^{m \ell} e_{\ell}^{b} .
\end{aligned}
$$

Let us remind that only $p+1$ components of $\lambda^{\underline{a}}$ are independent, as expressed in (B.24), and that the anti-symmetric parts of $\Omega_{a b}$ and $\Omega_{a^{\prime} b^{\prime}}$ remain undetermined.

In summary we have the primary constraints (with their number in parenthesis)

$$
\begin{array}{rlrl}
\phi_{\underline{a}} & =p_{\underline{a}}+\kappa^{\prime} e e_{b}^{0} \Phi_{\underline{a}}{ }^{b}=0, & (D), \\
\phi_{\underline{\underline{a}}}=\Pi^{\underline{a}} \underline{\underline{b}}=0, & \left(D^{2}\right), \\
\phi \underline{a b}=p \frac{a b}{\gamma}=0, & \left(\frac{D(D+1)}{2}\right),
\end{array}
$$

and the secondary constraints

$$
\begin{array}{rlrl}
\chi^{\underline{a b}}=\frac{1}{2}\left(\eta \frac{\underline{c}}{\underline{d}} \Phi_{\underline{\underline{c}}}^{\underline{\underline{a}}} \Phi_{\underline{\underline{d}}}^{\underline{b}}-\eta^{\underline{a b}}\right)=0, & & \left(\frac{(D(D+1)}{2}\right), \\
\chi_{a b}^{\gamma}=\gamma_{a b}-\kappa \eta_{a b}=0, & & \left(\frac{(p+1)(p+2)}{2}\right), \\
\chi_{a^{\prime} b}^{\gamma}=\gamma_{a^{\prime} b}=0, & & (p+1)(D-(p+1)), \\
\chi_{a^{\prime} b^{\prime}}^{\gamma}=\gamma_{a^{\prime} b^{\prime}}=0, & & (p-(p+1))(D-p) \\
\chi_{m}{ }^{b^{\prime}}=\partial_{m} x^{\underline{a}} \Phi_{\underline{a}}{ }^{b^{\prime}}=0, & (p+1))) .
\end{array}
$$

The conditions for the Hamiltonian multipliers are Eq.(B.32) for $\lambda_{\underline{a b}}^{\gamma}$

$$
\lambda_{\underline{a b}}^{\gamma}=0, \quad\left(\frac{D(D+1)}{2}\right),
$$


Eq.(B.31) for $\Lambda=\Phi \Omega$

$$
\Omega \underline{a b}+\Omega \underline{b a}=0, \quad\left(\frac{D(D+1)}{2}\right),
$$

and Eq.(B.24) for $\lambda \underline{a}$

$$
\tilde{\lambda}^{b^{\prime}}=\lambda^{\underline{a}} \Phi_{\underline{a}}^{b^{\prime}}=0, \quad(D-(p+1)) .
$$

In addition there are $(p+1)(D-(p+1))$ linear relations (B.28)+(B.33) for $\lambda^{\underline{a}}$ and $\Omega_{b}^{b^{\prime}}$ which are solved for $\Omega_{b}^{b^{\prime}}$ as Eq.(B.38),

$$
\Omega_{a^{\prime}}^{b}=\left(\left(e e_{[d}^{m} e_{a]}^{0} \Phi_{\underline{a}}^{a} \partial_{m} \Phi_{\underline{c}}^{d} \Phi^{\underline{c}}{ }_{a^{\prime}}\right) \frac{\left(e e^{b 0}\right)}{\mathbf{g}}-\left(\partial_{m} \Phi_{a a^{\prime}}\right) \mathbf{g}^{m \ell} e_{\ell}^{b}\right) \lambda^{\underline{a}}
$$

The remaining arbitrary Hamiltonian multipliers are $\Omega_{a b}$ (in number $\frac{p(p-1)}{2}$ ), $\Omega_{a^{\prime} b^{\prime}}$ (in number $\frac{(D-(p+1))(D-p-2)}{2}$ ), and $\tilde{\lambda}^{a} \equiv \lambda \underline{a}_{\underline{a}}{ }^{a}$ (in number $p+1$ ). As expected, they correspond respectively to the local $S O(p+1), S O(D-(p+1))$ and Diff $_{p+1}$ gauge invariances.

\section{B.1 First and Second class constraints}

We classify the constraints into the second class constraints that reduce the dependent canonical variables and the first class constraints that generate the gauge transformations. The constraints (B.41) and (B.43)-(B.45) are the second class constraints that allow us to reduce the canonical pairs $\left(\gamma, p_{\gamma}\right)$,

$$
p \frac{a b}{\gamma}=0, \quad \gamma_{a b}=\kappa \eta_{a b}, \quad \gamma_{a b^{\prime}}=\gamma_{a^{\prime} b^{\prime}}=0
$$

The $D^{2}$ constraints (B.40) are rearranged $\operatorname{as}^{26}$

$$
K_{\underline{c d}} \equiv \Pi^{\underline{a}_{(\underline{c}}} \Phi_{\left.\underline{a}_{\underline{d}}\right)}=0, \quad J_{c d^{\prime}} \equiv \Pi^{\underline{a}}{ }_{[c} \Phi_{\left.\underline{a}_{d^{\prime}}\right]}=0,
$$

and

$$
J_{c d} \equiv \Pi \underline{a}_{[c} \Phi_{\left.\underline{a}_{d]}\right]}=0, \quad \quad J_{c^{\prime} d^{\prime}} \equiv \Pi_{{ }_{\left[c^{\prime}\right.}} \Phi_{\left.\underline{a}_{d^{\prime}}\right]}=0 .
$$

The $D$ constraints $\phi_{\underline{a}}$ in (B.39) are projected into

$$
\begin{aligned}
\tilde{\phi}_{b} & \equiv \phi_{\underline{a}} \Phi^{\underline{a}}{ }_{b}=\left(p_{\underline{a}} \Phi^{\underline{a}}{ }_{b}+\kappa e e_{b}^{0}\right)=0, & & (p+1), \\
\tilde{\phi}_{b^{\prime}} & \equiv \phi_{\underline{a}} \Phi^{\underline{a}_{b^{\prime}}}=p_{\underline{a}} \Phi^{\underline{a}_{b^{\prime}}}=0, & & (D-(p+1)) .
\end{aligned}
$$

The symmetric constraints $K_{\underline{a b}}$ 's, in number $\frac{D(D+1)}{2}$, are combined with the same number of $\chi^{\frac{a b}{b}}$ to form $\frac{D(D+1)}{2}$ pairs of second class constraints,

$$
\left.\left\{\chi^{\underline{a b}}, K_{\underline{c d}}\right\}=\delta^{\underline{a}}{ }_{(\underline{c}} \delta^{\underline{b}}{ }^{d}\right) .
$$

\footnotetext{
${ }^{26}$ Our convention of (anti-)symmetrizations are $A_{[a} B_{b]}=A_{a} B_{b}-A_{b} B_{a}$ and $A_{(a} B_{b)}=A_{a} B_{b}+A_{b} B_{a}$.
} 
The $(p+1)(D-(p+1))$ constraints $J_{a b^{\prime}}$ in (B.52) are paired with $p(D-(p+1))$ $\chi_{m}{ }^{a^{\prime}}$ in (B.46) and $(D-(p+1)) \tilde{\phi}_{b^{\prime}}$ in (B.55). They are actually second class pairs since they satisfy non singular set of Poisson brackets;

$$
\begin{aligned}
\left\{J_{c d^{\prime}}, \tilde{\phi}^{b^{\prime}}\right\} & ==\delta^{b^{\prime}} d^{\prime} p^{\underline{b}} \Phi_{\underline{b} c}=-\kappa e e_{c}{ }^{0} \delta^{b^{\prime}} d^{\prime}, \\
\left\{J_{c d^{\prime}}, \chi_{m}{ }^{b^{\prime}}\right\} & =\delta^{b^{\prime}}{ }_{d^{\prime}} \partial_{m} x^{\underline{\underline{b}}} \Phi_{\underline{b c}}=e_{m c} \delta^{b^{\prime}} d^{\prime} .
\end{aligned}
$$

The constraints appearing in the Hamiltonian (B.8) with arbitrary multipliers are the first class constraints. Let us start with the original Hamiltonian

$$
\mathcal{H}=\phi_{\underline{a}} \lambda^{\underline{a}}+\phi^{\underline{a}} \underline{\underline{b}} \Lambda_{\underline{a}} \underline{\underline{b}}+\phi \frac{a b}{\gamma} \lambda_{\underline{a b}}^{\gamma}
$$

and obtain the primary first class constraints present in this Hamiltonian (B.58) as the combinations of primary constraints that still keep arbitrary multipliers attached to them. In doing so we use the conditions of the multipliers (B.47)-(B.50) obtained from the consistency conditions of the constraints,

$$
\Omega^{T}+\Omega=0, \quad \lambda_{\underline{a b}}^{\gamma}=0, \quad \lambda^{\underline{a}} \Phi_{\underline{a}}^{b^{\prime}}=0,
$$

and (B.50). In addition to above conditions on the multipliers we repeatedly use the fact that product of two constraints vanish as strong equation in the Hamiltonian[29]

First we note using the completeness (B.37)

$$
\tilde{\lambda}^{a}=\lambda^{\underline{a}} \Phi_{\underline{a}}^{a}=\left(\frac{\eta^{a c}\left(e e_{c}^{0}\right)\left(e e_{b}^{0}\right)}{-\mathbf{g}}+e_{m}{ }^{a} \mathbf{g}^{m \ell} e_{\ell b}\right) \tilde{\lambda}^{b}=-\eta^{a c}\left(e e_{c}{ }^{0}\right) \hat{\lambda}^{\perp}+e_{m}{ }^{a} \hat{\lambda}^{m},
$$

where $\hat{\lambda}^{\perp}$ and $\hat{\lambda}^{m}$ are introduced by

$$
\hat{\lambda}^{\perp}=\frac{\left(e e_{b}^{0}\right)}{\mathbf{g}} \tilde{\lambda}^{b}, \quad \hat{\lambda}^{m}=\mathbf{g}^{m \ell} e_{\ell b} \tilde{\lambda}^{b} .
$$

The first term of (B.8) $\phi_{\underline{a}} \lambda^{\underline{a}}$ is written as

$$
\phi_{\underline{a}} \lambda^{\underline{a}}=\left(\phi_{\underline{a}} \Phi^{\underline{a}} b\right)\left(\lambda \underline{c}^{\underline{c}} \Phi^{b}\right)=\tilde{\phi}_{b} \tilde{\lambda}^{b}, \quad \tilde{\lambda}^{b}=\lambda^{\underline{c}} \Phi_{\underline{c}}{ }^{b}, \quad \tilde{\phi}_{b}=\phi_{\underline{a}} \Phi^{\underline{a}}{ }_{b} .
$$

Using the completeness (B.37)

$$
\begin{aligned}
\tilde{\phi}_{b} \tilde{\lambda}^{b}= & \left(p_{\underline{a}} \Phi^{\underline{a}}{ }_{a}+\kappa e e_{a}^{0}\right)\left(\frac{\eta^{a c}\left(e e_{c}^{0}\right)\left(e e_{b}{ }^{0}\right)}{-\mathbf{g}}+e_{m}{ }^{a} \mathbf{g}^{m \ell} e_{\ell b}\right) \tilde{\lambda}^{b} \\
= & \left(p_{\underline{a}} \Phi^{\underline{a}}{ }_{a}+\kappa e e_{a}{ }^{0}\right) \frac{\eta^{a c}\left(e e_{c}^{0}\right)\left(e e_{b}{ }^{0}\right)}{-\mathbf{g}} \tilde{\lambda}^{b}+\left(p_{\underline{a}} \Phi^{\underline{a}_{a}}+\kappa e e_{a}{ }^{0}\right) e_{m}{ }^{a} \mathbf{g}^{m \ell} e_{\ell b} \tilde{\lambda}^{b} \\
= & -\left(p_{\underline{a}} \Phi^{\underline{a}}{ }_{a}+\kappa e e_{a}{ }^{0}\right) \eta^{a c} \frac{\left(\left(p_{\underline{b}} \Phi^{\underline{b}}{ }_{c}+\kappa e e_{c}{ }^{0}\right)-\left(p_{\underline{b}} \Phi^{\underline{b}}{ }_{c}-\kappa e e_{c}{ }^{0}\right)\right)}{2 \kappa} \hat{\lambda}^{\perp} \\
& +\phi_{\underline{a}} \Phi^{\underline{a}}{ }_{a} \partial_{m} x^{\underline{b}} \Phi_{\underline{b}}{ }^{a} \mathbf{g}^{m \ell} e_{\ell b} \tilde{\lambda}^{b} .
\end{aligned}
$$

The last term becomes

$$
\begin{aligned}
\phi_{\underline{a}} \Phi \underline{a}_{a} \partial_{m} x^{\underline{b}} \Phi_{\underline{b}}{ }^{a} \hat{\lambda}^{m} & =\phi_{\underline{a}}\left(\delta \underline{a}_{\underline{b}}-\Phi \underline{a}_{a^{\prime}} \Phi_{\underline{b}}{ }^{a^{\prime}}\right) \partial_{m} x^{\underline{b}} \hat{\lambda}^{m}=\phi_{\underline{a}} \partial_{m} x^{\underline{a}} \hat{\lambda}^{m} \\
& =\left(p_{\underline{a}}+\kappa e e_{c}{ }^{0} \Phi_{\underline{a}}{ }^{c}\right) \partial_{m} x^{\underline{a}} \hat{\lambda}^{m}=p_{\underline{a}} \partial_{m} x^{\underline{a}} \hat{\lambda}^{m} \equiv \mathcal{H}_{m}^{1} \hat{\lambda}^{m} .
\end{aligned}
$$


where the square of constraint term $\left(\phi_{\underline{a}} \Phi \underline{a}_{a^{\prime}}\right)\left(\partial_{m} x^{\underline{b}} \Phi_{\underline{b}}{ }^{\prime}\right)$ has been dropped from second to third equation ${ }^{27}$. In the last second line of (B.63) we omit the square of constraint $\phi_{\underline{a}}^{2}$ term and

$$
\begin{aligned}
& \frac{1}{2 \kappa}\left(p_{\underline{a}} \Phi^{\underline{a}}{ }_{a}+\kappa e e_{a}{ }^{0}\right) \eta^{a c}\left(p_{\underline{b}} \Phi^{\underline{b}}{ }_{c}-\kappa e e_{c}{ }^{0}\right)=\frac{1}{2 \kappa}\left(p_{\underline{a}}\left(\eta^{\underline{a b}}-\Phi^{\underline{a}} a^{\prime} \Phi^{\underline{b} a^{\prime}}\right) p_{\underline{b}}-\kappa^{2} e^{2} g^{00}\right) \\
= & \frac{1}{2 \kappa}\left(p_{\underline{a}} \eta{ }^{\underline{a} \underline{b}} p_{\underline{b}}+\kappa^{2} \mathbf{g}\right) \equiv \mathcal{H}_{\perp}^{1}, \quad g_{m n}=\eta_{\underline{a b}} \partial_{m} x^{\underline{a}} \partial_{n} x^{\underline{b}},
\end{aligned}
$$

where again square of constraint terms $\left(p_{\underline{a}} \Phi_{a^{\prime}}\right)\left(\Phi_{\underline{b}}{ }^{\prime} p^{\underline{b}}\right)=\left(\phi_{\underline{a}} \Phi_{a_{a^{\prime}}}\right)\left(\Phi_{\underline{b}}{ }^{a^{\prime}} \phi^{\underline{b}}\right)$ have also been dropped from the second to the third equation. We arrive at

$$
\phi_{\underline{a}} \lambda^{\underline{a}}=\hat{\lambda}^{\perp} \mathcal{H}_{\perp}^{1}+\hat{\lambda}^{m} \mathcal{H}_{m}^{1},
$$

where

$$
\mathcal{H}_{\perp}^{1}=\frac{1}{2 \kappa}\left(\eta \eta^{\underline{a b}} p_{\underline{a}} p_{\underline{b}}+\kappa^{2} \mathbf{g}\right), \quad \mathcal{H}_{m}^{1}=p_{\underline{a}} \partial_{m} x^{\underline{a}} .
$$

The second term of (B.8) is

$$
\phi^{\underline{a}} \underline{\underline{a}} \Lambda_{\underline{a}} \underline{b}=\Pi \underline{a}_{\underline{b}} \Phi_{\underline{a c}} \Omega^{\underline{c} \underline{b}}=\frac{1}{2} J_{a b} \Omega^{b a}+\frac{1}{2} J_{a^{\prime} b^{\prime}} \Omega^{b^{\prime} a^{\prime}}+J_{b}{ }^{a^{\prime}} \Omega_{a^{\prime}}{ }^{b},
$$

where we have used the anti-symmetry of $\Omega$. Note that $\Omega^{b a}$ and $\Omega^{b^{\prime} a^{\prime}}$ are arbitrary while $\Omega_{a^{\prime}}{ }^{b}$ is given in (B.50). Let us write $J_{b}{ }^{a^{\prime}} \Omega_{a^{\prime}}{ }^{b}$ as a linear combination of $\hat{\lambda}^{\perp}$ and $\hat{\lambda}^{m}$. To do this we use (B.60) and

$$
\begin{aligned}
e e_{d}{ }^{m} & =\frac{-1}{(p-1) !} \epsilon_{d a_{1} \ldots a_{p}} \epsilon^{m 0 m_{2} \ldots m_{p}} e_{0}{ }^{a_{1}} e_{m_{2}}{ }^{a_{2}} \ldots e_{m_{p}}{ }^{a_{p}}=e e_{[d}{ }^{m} e_{\left.a_{0}\right]}{ }^{0} \lambda^{\underline{a}} \Phi_{\underline{a}}{ }^{a_{0}} \\
& =-e e_{[d}^{m} e_{a]}^{0}\left(e e^{a 0}\right) \hat{\lambda}^{\perp}+e e_{[d}{ }^{m} e_{\left.a_{0}\right]}{ }^{0} \Phi_{\underline{a}}{ }^{a_{0}}\left(\partial_{\ell} x^{\underline{a}} \hat{\lambda}^{\ell}\right)
\end{aligned}
$$

and take into account that in (B.50) $\Omega_{a^{\prime}}{ }^{b}$ is expressed in terms of $\hat{\lambda}^{\perp}$ and $\hat{\lambda}^{m}$. After some work we arrive at

$$
\begin{aligned}
J_{b}{ }^{a^{\prime}} \Omega_{a^{\prime}}{ }^{b} & =\left(\Pi \underline{\underline{a}}_{\underline{b}} \partial_{m} \Phi_{\underline{a}} \underline{b}+\frac{1}{2} J_{a b}\left(\Phi^{\underline{c} a} \partial_{m} \Phi_{\underline{c}}^{b}\right)+\frac{1}{2} J^{a^{\prime} b^{\prime}}\left(\Phi_{a^{\prime}} \partial_{m} \Phi_{b^{\prime}}\right)\right) \hat{\lambda}^{m} \\
& +\hat{\lambda}^{\perp} J^{b a^{\prime}}\left(e e_{[d}{ }^{m} e_{b]}{ }^{0}\right)\left(\partial_{m} \Phi_{\underline{c}}{ }^{d} \Phi^{\underline{c}} a^{\prime}\right) .
\end{aligned}
$$

In summary the Hamiltonian is written as

$$
\mathcal{H}=\hat{\lambda}^{\perp} \mathcal{H}_{\perp}+\hat{\lambda}^{m} \mathcal{H}_{m}+\frac{1}{2} J_{a b} \tilde{\Omega}^{b a}+\frac{1}{2} J_{a^{\prime} b^{\prime}} \tilde{\Omega}^{b^{\prime} a^{\prime}}
$$

with the first class constraints

$$
\begin{aligned}
\mathcal{H}_{\perp} & =\frac{1}{2 \kappa}\left(\eta{ }^{\underline{a b}} p_{\underline{a}} p_{\underline{b}}+\kappa^{2} \mathbf{g}\right)+J^{b a^{\prime}}\left(e e_{[d}^{m} e_{b]}^{0}\right)\left(\partial_{m} \Phi_{\underline{c}}{ }^{d} \Phi^{\underline{c}} a^{\prime}\right), \\
\mathcal{H}_{m} & =p_{\underline{a}} \partial_{m} x^{\underline{a}}+\Pi \underline{a}_{\underline{b}} \partial_{m} \Phi_{\underline{a}} \underline{\underline{b}} \\
J_{a b} & =\Pi^{\underline{c}}{ }_{[a} \Phi_{\underline{c} b]}, \\
J_{a^{\prime} b^{\prime}} & =\Pi \underline{\underline{c}}_{\left[a^{\prime}\right.} \Phi_{\left.\underline{c} b^{\prime}\right]}
\end{aligned}
$$

\footnotetext{
${ }^{27}$ In (B.64) use has been made also of the secondary constraints (B.42). One can check that their contribution cancels out in the final expressions.
} 
and with a redefinition of arbitrary functions,

$$
\tilde{\Omega}^{b a}=\Omega^{b a}+\left(\Phi^{\underline{c} a} \partial_{m} \Phi_{\underline{c}}^{b}\right) \hat{\lambda}^{m}, \quad \tilde{\Omega}^{b^{\prime} a^{\prime}}=\Omega^{b^{\prime} a^{\prime}}+\left(\Phi^{\underline{c} a^{\prime}} \partial_{m} \Phi_{\underline{c}}^{b^{\prime}}\right) \hat{\lambda}^{m} .
$$

The $\frac{(p+1) p}{2}$ constraints $J_{a b}$ and the $\frac{(D-(p+1))(D-p-2)}{2}$ constraints $J_{a^{\prime} b^{\prime}}$ are respectively the $S O(1, p)$ and $S O(D-(p+1))$ local generators for the $\Phi$ variables, satisfying the algebras,

$$
\begin{aligned}
\left\{J_{a b}(\xi), J_{c d}\left(\xi^{\prime}\right)\right\} & =\left(\eta_{b[c} J_{a d]}(\xi)-\eta_{a[c} J_{b d]}(\xi)\right) \delta^{p}\left(\xi-\xi^{\prime}\right), \\
\left\{J_{a^{\prime} b^{\prime}}(\xi), J_{c^{\prime} d^{\prime}}\left(\xi^{\prime}\right)\right\} & =\left(\eta_{b^{\prime}\left[c^{\prime}\right.} J_{\left.a^{\prime} d^{\prime}\right]}(\xi)-\eta_{a^{\prime}\left[c^{\prime}\right.} J_{\left.b^{\prime} d^{\prime}\right]}(\xi)\right) \delta^{p}\left(\xi-\xi^{\prime}\right) .
\end{aligned}
$$

The Diff $p+1$ generators $\mathcal{H}_{\perp}$ and $\mathcal{H}_{m}{ }^{28}$ are multiplied by local functions as

$$
\mathcal{H}_{\perp}[h]=\int d^{p} \xi h(\xi) \mathcal{H}_{\perp}(\xi), \quad \mathcal{H}_{m}\left[f^{m}\right]=\int d^{p} \xi f^{m}(\xi) \mathcal{H}_{m}(\xi),
$$

and satisfy a closed algebra as

$$
\begin{aligned}
\left\{\mathcal{H}_{\perp}[h], \mathcal{H}_{m}\left[f^{m}\right]\right\} & =\mathcal{H}_{\perp}\left[h \partial_{m} f^{m}-f^{m} \partial_{m} h\right], \\
\left\{\mathcal{H}_{\ell}\left[h^{\ell}\right], \mathcal{H}_{m}\left[f^{m}\right]\right\} & =\mathcal{H}_{\ell}\left[h^{m} \partial_{m} f^{\ell}-f^{m} \partial_{m} h^{\ell}\right], \\
\left\{\mathcal{H}_{\perp}[f], \mathcal{H}_{\perp}[h]\right\} & =\int d \sigma \mathbf{g g}^{\ell m}\left(f \partial_{m} h-h \partial_{m} f\right) \\
& \times\left(\mathcal{H}_{\ell}-\frac{1}{2}\left(J^{a b}\left(\Phi^{\underline{b}} \partial_{\ell} \Phi_{\underline{b} a}\right)+J^{a^{\prime} b^{\prime}}\left(\Phi_{\underline{b} b^{\prime}} \partial_{\ell} \Phi^{\underline{b}} a^{\prime}\right)\right)\right) .
\end{aligned}
$$

The last equation means that the commutators of two local Diff transformations generated by $\mathcal{H}_{\perp}$ results in a local transverse Diff $\mathcal{H}_{m}$ and associated local Lorentz transformations $S O(1, p)$ and $S O(D-(p+1))$. Finally

$$
\begin{aligned}
\left\{\mathcal{H}_{m}\left[f^{m}\right], J_{a b}(\xi)\right\} & =-\partial_{m}\left(f^{m} J_{a b}(\xi)\right), \quad\left\{\mathcal{H}_{m}\left[f^{m}\right], J_{a^{\prime} b^{\prime}}(\xi)\right\}=-\partial_{m}\left(f^{m} J_{a^{\prime} b^{\prime}}(\xi)\right), \\
\left\{\mathcal{H}_{\perp}[h], J_{a b}(\xi)\right\} & =\left\{\mathcal{H}_{\perp}[h], J_{a^{\prime} b^{\prime}}(\xi)\right\}=0
\end{aligned}
$$

showing that $\left(\mathcal{H}_{\perp}, \mathcal{H}_{m}, J_{a b}, J_{a^{\prime} b^{\prime}}\right)$ forms a first class constraint set.

Let us consider the example of the string, where we have $a, b=0,1, m=\ell=1$. The arbitrary functions $\hat{\lambda}^{\perp}$ and $\hat{\lambda}^{m},(m=1)$ have the familiar forms,

$$
\hat{\lambda}^{\perp}=\frac{e}{\mathbf{g}}=\frac{\sqrt{-g}}{g_{11}}, \quad \tilde{\lambda}^{1}=\mathbf{g}^{11} g_{10}=\frac{g_{10}}{g_{11}} .
$$

$\mathcal{H}_{\perp}$ becomes ("prime" means $\xi^{1}$ derivative)

$$
\mathcal{H}_{\perp}=\frac{1}{2 \kappa}\left(\eta^{\underline{a b}} p_{\underline{a}} p_{\underline{b}}+\kappa^{2} x_{\underline{a}}^{\prime 2}\right)+J^{b a^{\prime}} \epsilon_{d b}\left(\Phi_{\underline{c}}^{\prime d} \Phi^{\underline{c}} a^{\prime}\right),
$$

where $\left(e e_{[d}^{1} e_{b]}^{0}\right)=\epsilon_{d b}$ and the second term commutes with the first term. One can easily check the closure of the algebra for the string case,

$$
\begin{gathered}
\left\{\mathcal{H}_{\perp}[f], \mathcal{H}_{\perp}[h]\right\}=\mathcal{H}_{1}\left[f h^{\prime}-f^{\prime} h\right]-\frac{1}{2} \int d \sigma\left(f h^{\prime}-f^{\prime} h\right)\left(J^{a b}\left(\Phi^{\underline{b}} \Phi_{\underline{b} a}^{\prime}\right)+J^{a^{\prime} b^{\prime}}\left(\Phi_{\underline{b} b^{\prime}} \Phi^{\prime} \underline{b}_{a^{\prime}}\right)\right), \\
\left\{\mathcal{H}_{1}[f], \mathcal{H}_{\perp}[h]\right\}=\mathcal{H}_{\perp}\left[f h^{\prime}-f^{\prime} h\right], \quad\left\{\mathcal{H}_{1}[f], \mathcal{H}_{1}[h]\right\}=\mathcal{H}_{1}\left[f h^{\prime}-f^{\prime} h\right] .
\end{gathered}
$$

\footnotetext{
${ }^{28}$ Regarding $p$-dimensional world-sheet integrations, $\mathcal{H}_{\perp}$ in (B.72) is a density of weight 2 and the multiplier $\hat{\lambda}^{\perp}$ is a density of weight $-1, \mathcal{H}_{m}$ is a vector density of weight 1 and the multipliers $\hat{\lambda}^{m}$ behave as a vector. All this guarantees that (B.71) is a density of weight 1 as expected.
} 


\section{B.2 Reduced phase space and Gauge fixing}

The second class constraints are used to reduce the phase space variables. In order to find them explicitly it is convenient to rewrite the $D^{2}$ variables $\Phi_{\underline{a}}^{\underline{b}}$ in terms of same numbers of coordinates

$$
\begin{array}{rlll}
\phi_{a}{ }^{b} ; & (p+1)^{2}, & \varphi_{a}{ }^{b^{\prime}} ; & (p+1)(D-(p+1)), \\
\tilde{\varphi}_{a}^{b^{\prime}} ; & (p+1)(D-(p+1)), & \phi_{a^{\prime}}{ }^{b^{\prime}} ; & (D-(p+1))^{2} .
\end{array}
$$

as

$$
\Phi_{\underline{a}}^{\underline{b}}=\left(\begin{array}{cc}
B_{1 a}{ }^{c} & \tilde{\varphi}_{a}^{c^{\prime}} B_{2 c^{\prime}} d^{\prime} \\
-\varphi^{d}{ }_{a^{\prime}} B_{1 d}{ }^{c} & B_{2 a^{\prime}} d^{\prime}
\end{array}\right)\left(\begin{array}{cc}
\phi_{c}{ }^{b} & 0 \\
0 & \phi_{d^{\prime}}{ }^{b^{\prime}}
\end{array}\right)
$$

where $B_{1}$ and $B_{2}$ are symmetric matrices defined by

$$
\left(\left(B_{1}\right)^{-2}\right)_{a}^{b}=\left(\delta_{a}{ }^{b}+\varphi_{a}{ }^{c^{\prime}} \varphi^{b}{ }_{c^{\prime}}\right), \quad\left(\left(B_{2}\right)^{-2}\right)_{a^{\prime}}{ }^{b^{\prime}}=\left(\delta_{a^{\prime}}{ }^{b^{\prime}}+\tilde{\varphi}^{c}{ }_{a^{\prime}} \tilde{\varphi}_{c}{ }^{b^{\prime}}\right) .
$$

In terms of new variables (B.81) the orthonormality constraints (B.42) are written as

$$
\phi_{a}{ }^{c} \phi^{b}{ }_{c}=\left(\phi \phi^{T}\right)_{a}^{b}=\delta_{a}{ }^{b}, \quad \phi_{c^{\prime}}{ }^{a^{\prime}} \phi_{b^{\prime}}^{c^{\prime}}=\left(\phi^{\prime T} \phi^{\prime}\right)^{a^{\prime}}{ }_{b^{\prime}}=\delta^{a^{\prime}}{ }_{b^{\prime}}, \quad \tilde{\varphi}_{a}^{b^{\prime}}=\varphi_{a}{ }^{b^{\prime}} .
$$

It means that $\phi_{a}{ }^{b}$ is an element of $S O(p, 1)$ and $\phi_{a^{\prime}}{ }^{b^{\prime}}$ is an element of $S O(D-(p+1))$.

One can adapt the canonical variables to the redefinition (B.82) of the $\Phi$ configuration variables. Indeed, making a canonical transformation $\Phi \rightarrow\left(\phi, \phi^{\prime}, \varphi, \tilde{\varphi}\right)$ generated by

$$
W\left(\Pi, \phi, \phi^{\prime}, \varphi, \tilde{\varphi}\right)=\Pi \underline{a}_{\underline{b}} \Phi_{\underline{a}} \underline{b}\left(\phi, \phi^{\prime}, \varphi, \tilde{\varphi}\right),
$$

we obtain the new momenta as $\pi_{\phi}=\frac{\partial W}{\partial \phi}$, etc. The primary constraints $\Pi=0$ in (B.40) become, in terms of the new momenta, as

$$
\pi_{\phi}=\pi_{\phi^{\prime}}=\pi_{\varphi}=\pi_{\tilde{\varphi}}=0 .
$$

The gauge $S O(p+1)$ and $S O(D-(p+1))$ degrees of freedom can be eliminated by imposing gauge fixing constraints for the $S O(p+1)$ and $S O(D-(p+1))$ matrices. The simplest choice for this gauge fixing is

$$
\phi_{a}^{b}=\delta_{a}^{b}, \quad \phi_{a^{\prime}}{ }^{b^{\prime}}=\delta_{a^{\prime}}{ }^{b^{\prime}} .
$$

Note now that $\pi_{\tilde{\varphi}}=0$ and $\tilde{\varphi}-\varphi=0$ are the second class constraints to eliminate the $(p+1)(D-(p+1))$ canonical pairs

$$
\left(\tilde{\varphi}_{a}{ }^{b^{\prime}}, \pi_{\tilde{\varphi}_{b^{\prime}}}^{a}\right)=\left(\varphi_{a}{ }^{b^{\prime}}, 0\right) .
$$

Using them $\Phi$ is expressed in terms of $\varphi$ only as in [10], see also [30],

$$
\Phi_{\underline{a}}^{\underline{b}}=\left(\begin{array}{cc}
B_{1 a}^{b} & \varphi_{a}{ }^{c^{\prime}} B_{2 c^{c^{\prime}}}{ }^{b^{\prime}} \\
-\varphi^{c}{ }_{a^{\prime}} B_{1 c}{ }^{b} & B_{2 a^{\prime}} b^{\prime}
\end{array}\right) .
$$

The $\varphi$ 's are further determined by solving the second class constraints $\chi_{m}{ }^{a^{\prime}}=0$ and the $(D-(p+1))$ constraints $\tilde{\phi}^{a^{\prime}}=0$. In the gauge (B.87) they are

$$
\chi_{m}{ }^{a^{\prime}}=\left(\partial_{m} x^{a} \varphi_{a}^{b^{\prime}}+\partial_{m} x^{b^{\prime}}\right) B_{2 b^{\prime}}{ }^{\prime}=0,
$$




$$
\tilde{\phi}^{a^{\prime}}=\left(p^{a} \varphi_{a}^{b^{\prime}}+p^{b^{\prime}}\right) B_{2 b^{\prime}}{ }^{\prime}=0 .
$$

They are combined with $\pi_{\varphi}=0$ in (B.86) to form $(p+1)(D-(p+1))$ second class constraints pairs to eliminate $\varphi_{a}{ }^{b^{\prime}}$ in terms of $p_{a}$ and $\partial_{m} x^{\underline{a}}$ as in (2.43). Notice that $\varphi_{a}{ }^{b^{\prime}}$ are written as functions of the phase space variables of the $p$-brane.

Now all second class constraints are used to reduce the phase space to that of $\left(x^{\underline{a}}, p_{\underline{a}}\right)$. There remain the first class constraints $\mathcal{H}_{\perp}=\mathcal{H}_{m}=0$, and the first class Hamiltonian becomes

$$
\begin{gathered}
\mathcal{H}=\int d^{p} \xi\left(\hat{\lambda}^{\perp} \mathcal{H}_{\perp}^{1}+\hat{\lambda}^{m} \mathcal{H}_{m}^{1}\right), \\
\mathcal{H}_{\perp}^{1}=\frac{1}{2 \kappa}\left(\eta^{\underline{a b}} p_{\underline{a}} p_{\underline{b}}+\kappa^{2} \mathbf{g}\right), \quad \mathcal{H}_{m}^{1}=p_{\underline{a}} \partial_{m} x^{\underline{a}} .
\end{gathered}
$$

The action of $p$-brane obtained from the non-linear realization in the reduced space leads to the canonical action of a Dirac-Nambu-Goto $p$-brane action in configuration space.

\section{References}

[1] M. Luscher and P. Weisz, "String excitation energies in SU(N) gauge theories beyond the free-string approximation," JHEP 0407 (2004) 014 [hep-th/0406205].

[2] J. Polchinski and A. Strominger, "Effective string theory," Phys. Rev. Lett. 67, 1681 (1991).

[3] O. Aharony and M. Dodelson, "Effective String Theory and Nonlinear Lorentz Invariance," arXiv:1111.5758 [hep-th].

[4] M. Billo, M. Caselle, F. Gliozzi, M. Meineri and R. Pellegrini, "The Lorentzinvariant boundary action of the confining string and its universal contribution to the inter-quark potential," arXiv:1202.1984 [hep-th].

[5] S. Dubovsky, R. Flauger and V. Gorbenko, "Effective String Theory Revisited," arXiv:1203.1054 [hep-th].

[6] S. R. Coleman, J. Wess and B. Zumino, "Structure of phenomenological Lagrangians. 1," Phys. Rev. 177 (1969) 2239; C. G. . Callan, S. R. Coleman, J. Wess and B. Zumino, "Structure of phenomenological Lagrangians. 2," Phys. Rev. 177 (1969) 2247.

[7] V.I. Ogievetsky, "Non-linear realizations of internal and spacetime symmetries", in: Proc. 10th Karpacz Winter School of Theoretical physics ( 1974).

[8] E. Ivanov and S. Krivonos, "N=1 D $=4$ supermembrane in the coset approach," Phys. Lett. B 453 (1999) 237 [Erratum-ibid. B 657 (2007) 269] [hepth/9901003].

[9] S. Bellucci, E. Ivanov and S. Krivonos, "AdS / CFT equivalence transformation," Phys. Rev. D 66 (2002) 086001 [Erratum-ibid. D 67 (2003) 049901] [hepth/0206126].

[10] J. Gomis, K. Kamimura and P. West, "The Construction Of Brane And Superbrane Actions Using Non-linear Realisations," Class. Quant. Grav. 23 (2006) 7369 [arXiv:hep-th/0607057]. 
[11] J. Gomis, K. Kamimura and P. C. West, "Diffeomorphism, kappa transformations and the theory of non-linear realisations," JHEP 0610 (2006) 015 [hepth/0607104].

[12] E. A. Ivanov and V. I. Ogievetsky, "The Inverse Higgs Phenomenon in Nonlinear Realizations," Teor. Mat. Fiz. 25 (1975) 164.

[13] I. N. McArthur, "Nonlinear realizations of symmetries and unphysical Goldstone bosons," JHEP 1011 (2010) 140 [arXiv:1009.3696 [hep-th]].

[14] I. N. McArthur, "Kappa symmetry of Green-Schwarz actions in coset superspaces," Nucl. Phys. B 573, 811 (2000) [hep-th/9908045].

[15] R. .Casalbuoni, J. Gomis, K. Kamimura and G. Longhi, "Space-time Vector Supersymmetry and Massive Spinning Particle," JHEP 0802 (2008) 094 [arXiv:0801.2702 [hep-th]].

[16] G. W. Gibbons, J. Gomis and C. N. Pope, "Deforming the Maxwell-Sim Algebra," Phys. Rev. D 82 (2010) 065002 [arXiv:0910.3220 [hep-th]].

[17] J. P. Gauntlett, K. Itoh and P. K. Townsend, "Superparticle With Extrinsic Curvature," Phys. Lett. B 238 (1990) 65.

[18] J. P. Gauntlett, J. Gomis and P. K. Townsend, "Particle Actions As Wesszumino Terms For Space-time (super)symmetry Groups," Phys. Lett. B 249 (1990) 255.

[19] M. Dunajski and G. Gibbons, "Cosmic Jerk, Snap and Beyond," Class. Quant. Grav. 25 (2008) 235012 [arXiv:0807.0207 [gr-qc]].

[20] J. G. Russo and P. K. Townsend, "Relativistic Kinematics and Stationary Motions," J. Phys. A A 42 (2009) 445402 [arXiv:0902.4243 [hep-th]].

[21] E. Ramos, "Three-dimensional strings. 1. Classical theory," hep-th/9703117. E. Ramos, Nucl. Phys. B 519 (1998) 435 [hep-th/9709131].

[22] T. Curtright, "Strings on a plane," Phys. Lett. B 693 (2010) 477.

[23] S. Bonanos and J. Gomis, "A note on the Chevalley-Eilenberg Cohomology for the Galilei and Poincare Algebras," J. Phys. A 42 (2009) 145206 [arXiv:0808.2243 [hep-th]].

[24] F. Gliozzi and M. Meineri, "Lorentz completion of effective string (and p-brane) action," JHEP 1208 (2012) 056 [arXiv:1207.2912 [hep-th]].

[25] M. Bando, T. Kugo and K. Yamawaki, "Nonlinear Realization and Hidden Local Symmetries," Phys. Rept. 164 (1988) 217.

[26] I. A. Bandos and A. A. Zheltukhin, "Green-Schwarz superstrings in spinor moving frame formalism," Phys. Lett. B 288 (1992) 77.

[27] I. A. Bandos, D. P. Sorokin, M. Tonin, P. Pasti and D. V. Volkov, "Superstrings and supermembranes in the doubly supersymmetric geometrical approach," Nucl. Phys. B 446 (1995) 79 [hep-th/9501113].

[28] T. Curtright, G. Ghandour and C. K. Zachos, "Classical Dynamics Of Strings With Rigidity," Phys. Rev. D 34 (1986) 3811.

[29] P. A. M. Dirac, "Generalized Hamiltonian dynamics," Can. J. Math. 2, 129 (1950). 
[30] T. Kugo and K. Yoshioka, "Probing extra dimensions using Nambu-Goldstone bosons," Nucl. Phys. B 594 (2001) 301 [hep-ph/9912496].

[31] R. L. Arnowitt, S. Deser and C. W. Misner, "The dynamics of general relativity," in Gravitation: an introduction to current research, Louis Witten ed. (Wilew 1962), 1962. arXiv:gr-qc/0405109.

[32] J. Gomis and H. Ooguri, "Nonrelativistic closed string theory," J. Math. Phys. 42 (2001) 3127 [hep-th/0009181].

[33] U. H. Danielsson, A. Guijosa and M. Kruczenski, "IIA/B, wound and wrapped," JHEP 0010 (2000) 020 [hep-th/0009182].

[34] J. Brugues, T. Curtright, J. Gomis and L. Mezincescu, "Non-relativistic strings and branes as non-linear realizations of Galilei groups," Phys. Lett. B 594 (2004) 227 [hep-th/0404175].

[35] J. Gomis, J. Gomis and K. Kamimura, "Non-relativistic superstrings: A New soluble sector of AdS(5) x S**5," JHEP 0512 (2005) 024 [hep-th/0507036].

[36] J. Brugues, J. Gomis and K. Kamimura, "Newton-Hooke algebras, nonrelativistic branes and generalized pp-wave metrics," Phys. Rev. D 73 (2006) 085011 [hep-th/0603023].

[37] G. W. Gibbons, J. Gomis and C. N. Pope, "General very special relativity is Finsler geometry," Phys. Rev. D 76 (2007) 081701 [arXiv:0707.2174 [hep-th]].

[38] E. Ramos and J. Roca, "Extended gauge invariance in geometrical particle models and the geometry of W symmetry," Nucl. Phys. B 452, 705 (1995) [hepth/9504071].

[39] M. S. Plyushchay, "The Model of relativistic particle with torsion," Nucl. Phys. B 362 (1991) 54.

[40] J. F. Schonfeld, "A Mass Term for Three-Dimensional Gauge Fields," Nucl. Phys. B 185 (1981) 157.

[41] L. Mezincescu and P. K. Townsend, "Semionic Supersymmetric Solitons," J. Phys. A A 43 (2010) 465401 [arXiv:1008.2775 [hep-th]]. 